\title{
Electrically Charged Topological Solitons
}

\author{
J.F. Gomes, E. P. Gueuvoghlanian, G.M. Sotkov and A.H. Zimerman \\ Instituto de Física Teórica - IFT/UNESP \\ Rua Pamplona 145 \\ 01405-900, São Paulo - SP, Brazil \\ jfg@ift.unesp.br, gueuvogh@ift.unesp.br, sotkov@ift.unesp.br, zimerman@ift.unesp.br
}

\begin{abstract}
Two new families of T-Dual integrable models of dyonic type are constructed. They represent specific $A_{n}^{(1)}$ singular Non-Abelian Affine Toda models having $U(1)$ global symmetry. Their 1-soliton spectrum contains both neutral and $U(1)$ charged topological solitons sharing the main properties of 4-dimensional Yang-Mills-Higgs monopoles and dyons. The semiclassical quantization of these solutions as well as the exact counterterms and the coupling constant renormalization are studied.
\end{abstract}




\section{Introduction}

In search for new nonperturbative methods of quantization of four dimensional (nonsupersymmetric) $S U(n+1)$ QCD (and its bosonic part- the Yang-Mills-Higgs (YMH) model ), the relationship between 4-D selfdual Yang-Mills (SDYM) theories and certain 2-D integrable models (IM's) [1] deserves special attention. As it is well known the dimensional (and symmetries) reduction of 4-D SDYM to lower dimensional ( $D=1,2,3)$ IM's (see [2] for a review) provides effective methods for the construction of a large class of exact (classical) solutions of the 4-D gauge theory, including spherical symmetric monopoles [3], [4], instantons [5], domain walls (DW) [6], [7] etc. Among them the solitons of relativistic 2-D IM's are of particular interest:

- They are expected to describe (at least in large n limit ) DW's solutions of 4-D $S U(n+1)$ YMH theory;

- Their exact quantization is known to be related to the centerless affine quantum group $U_{q}\left(A_{n}^{(1)}\right)\left(\right.$ or $\left.U_{q}\left(A_{2 n}^{(2)}\right), U_{q}\left(A_{2 n-1}^{(2)}\right)\right)$ [8].

Whether $U_{q}\left(A_{n}^{(1)}\right)$ (or its dual ) takes place in the description of the quantum DW's and the other "strong coupling" solutions of $S U(n+1)$ YMH theory - monopoles, dyons and strings - is an open problem. There exists however a hint in favour of such conjectute, namely: The space of classical solutions of 4-D $S U(n+1)$ SDYM (and BPS solutions of YMH) has the affine $\hat{S U}(n+1)$ loop algebra as dynamic symmetry [9], which coincides with the classical limit $q \rightarrow 1(h \rightarrow 0)$ of the quantum soliton symmetry group $U_{q}\left(A_{n}^{(1)}\right)$ [

The domain walls that appear in the string (i.e. D-branes ) description of 4-D gauge theories [6], [7] manifest properties a bit more involved than the simplest topological solitons of say, sine-Gordon or abelian affine Toda models. For instance, they may carry certain $U(1)$ charges or require nonmaximal breaking of $S U(n+1)$ gauge group, say $S U(k) \otimes U(1)^{n-k+1}$ (i.e. $k$-coincident D-branes) [7]. The question arises whether one can find 2-D IM's whose soliton solutions possess all DW's characteristics. The problem we address in the present paper is to construct such dyonic integrable models t admiting $U(1)$-charged topological solitons and CPT-violating term. We shall study the solitonic spectrum (classical and quantum ) of the following new family of (CPT-noninvariant) $A_{n}^{(1)}$ dyonic IM's $(n>1)$

$$
\begin{gathered}
\mathcal{L}_{a}=\frac{1}{2} \sum_{i, k=1}^{n-1} \eta_{i k} \partial \varphi_{i} \bar{\partial} \varphi_{k}+\frac{\partial \chi \bar{\partial} \psi e^{-\beta \varphi_{1}}}{\Delta}-V_{a} \\
V_{a}=\frac{m^{2}}{\beta^{2}}\left(\sum_{i=1}^{n-1} e^{\beta\left(\varphi_{i-1}+\varphi_{i+1}-2 \varphi_{i}\right)}+e^{\beta\left(\varphi_{1}+\varphi_{n-1}\right)}\left(1+\beta^{2} \psi \chi e^{-\beta \varphi_{1}}\right)-n\right)
\end{gathered}
$$

\footnotetext{
${ }^{1}$ The Sine Gordon (SG) and the $\hat{G}_{n}$-abelian affine Toda model are examples of magnetic type IM having neutral topological solitons. The Complex Sine Gordon ( or Lund-Regge) model [10, [11, 12] and its homogeneous space SG generalizations [13] represent the electric type IM's, whose solitons are $U(1)$ (or $\left.U(1)^{r}\right)$ charged but nontopological.
} 
where $\eta_{i k}=2 \delta_{i, k}-\delta_{i, k-1}-\delta_{i, k+1}, \quad i, k=1, \cdots n-1, \Delta=1+\beta^{2} \frac{n+1}{2 n} \psi \chi e^{-\beta \varphi_{1}}, \varphi_{0}=\varphi_{n}=0$, $\partial=\partial_{t}+\partial_{x}, \bar{\partial}=\partial_{t}-\partial_{x}$ and $\beta^{2}=-\frac{2 \pi}{K}$.The IM's (1.1) are invariant under global $U(1)$ transformation,

$$
\psi^{\prime}=e^{-i a \beta^{2}} \psi, \quad \chi^{\prime}=e^{i a \beta^{2}} \chi, \quad \varphi_{i}^{\prime}=\varphi_{i}
$$

where $a$ is a real constant.They represent a specific mixture of the Lund-Regge model interacting with the $A_{n}^{(1)}$-abelian Toda theory. It is worthwhile to mention that the above dyonic models appear to be an appropriate integrable deformation of the recently constructed $V_{n+1^{-}}^{(1)}$ algebra minimal models [14] (i.e. the singular $A_{n}$-Non Abelian (NA) Toda theories):

$$
\mathcal{L}=\mathcal{L}_{\text {conf }}-\frac{m^{2}}{\beta^{2}} V_{\text {def }}, \quad V_{\text {def }}=e^{\beta\left(\varphi_{1}+\varphi_{n-1}\right)}\left(1+\beta^{2} \psi \chi e^{-\beta \varphi_{1}}\right)
$$

The CPT-violating term $e^{-\beta \varphi_{1}} \Delta^{-1} \epsilon^{\mu \nu} \partial_{\mu} \psi \partial_{\nu} \chi$ is hidden in the second term of (1.1)

$$
\frac{e^{-\beta \varphi_{1}}}{\Delta} \bar{\partial} \psi \partial \chi=\frac{e^{-\beta \varphi_{1}}}{\Delta}\left(g^{\mu \nu} \partial_{\mu} \psi \partial_{\nu} \chi+\epsilon^{\mu \nu} \partial_{\mu} \psi \partial_{\nu} \chi\right)
$$

It has the same origin as in the conformal theory $\mathcal{L}_{\text {conf }}$, i.e. the axial gauging of the $U(1)$ subgroup spanned by $\lambda_{1} \cdot H$. The CPT-invariant partner of (1.1) is the following vector type $A_{n}^{(1)}$-dyonic model:

$$
\begin{aligned}
\mathcal{L}_{v} & =\frac{1}{\beta^{2}}\left(\frac{1}{2} \sum_{i=1}^{n-1}\left[\partial \ln c_{i} \bar{\partial} \ln \left(c_{i} c_{i+1} \cdots c_{n-1}\right)+\bar{\partial} \ln c_{i} \partial \ln \left(c_{i} c_{i+1} \cdots c_{n-1}\right)\right]\right. \\
& \left.-\frac{1}{2} \frac{\partial A \bar{\partial} B+\partial B \bar{\partial} A}{1-A B}-\beta^{2} V_{v}\right) \\
V_{v}= & \frac{m^{2}}{\beta^{2}}\left(A c_{1}^{2} c_{2} \cdots c_{n-1}+\frac{B}{c_{1} c_{2} \cdots c_{n-2} c_{n-1}^{2}}+\frac{c_{2}}{c_{1}}+\frac{c_{3}}{c_{2}}+\cdots+\frac{c_{n-1}}{c_{n-2}}-n\right)
\end{aligned}
$$

It is obtained by vector gauging of the same $U(1)$ subgroup and appears to be T-dual to the " axial" model (1.1) [15. The ungauged integrable model giving rise to both models (1.1) and (1.3) has the following Lagrangean \&

$$
\begin{gathered}
\mathcal{L}_{u}=\frac{1}{2} \eta_{i k} \partial \varphi_{i} \bar{\partial} \varphi_{k}+\frac{(n+1)}{2 n} \partial R \bar{\partial} R+\partial \tilde{\chi} \bar{\partial} \tilde{\psi} e^{\beta\left(\frac{(n+1) R}{n}-\varphi_{1}\right)}-V_{u} \\
V_{u}=\frac{m^{2}}{\beta^{2}}\left(\sum_{i=1}^{n-1} e^{\beta\left(\varphi_{i-1}+\varphi_{i+1}-2 \varphi_{i}\right)}+e^{\beta\left(\varphi_{1}+\varphi_{n-1}\right)}\left(1+\beta^{2} \tilde{\psi} \tilde{\chi} e^{\beta\left(\frac{(n+1) R}{n}-\varphi_{1}\right)}\right)-n\right)
\end{gathered}
$$

The corresponding action $S_{u}$ is invariant under chiral $U(1)$ gauge transformations:

$$
R^{\prime}=R+w(z)+\bar{w}(\bar{z}), \quad \tilde{\psi}^{\prime}=e^{-\frac{\beta(n+1) w(z)}{n}} \tilde{\psi}, \quad \tilde{\chi}^{\prime}=e^{-\frac{\beta(n+1) \bar{w}(\bar{z})}{n}} \tilde{\chi}
$$

\footnotetext{
${ }^{2}$ Note that the $n=2$, (i.e. $\left.S L(3, R)\right)$ IM is nothing but the (thermal operator ) integrable perturbation of the Bershadsky-Polyakov $W_{3}^{(2)}$ minimal models [16].
} 
where $\psi=\tilde{\psi} e^{\frac{(n+1)}{2 n} R}, \chi=\tilde{\chi} e^{\frac{(n+1)}{2 n} R}$.

The zero curvature representation and the proof of the classical integrability of all models (1.1), (1.3) and (1.4), is derived in Sect. 2. Special attention is devoted to the discussion of discrete symmetries of (1.1) and (1.3). They are crucial in the definition of the vacuum lattice, the spectra of the topological charges $Q_{k}^{\text {top. }}$ and $Q_{\theta}$ as well as in the derivation of the first order 1-soliton equations in Sect.3. It is important to note that the first order system (3.7) has to be completed with a chain of algebraic relations (3.8) in order to determine a consistent vacua Backlund transformation. They represent 2-D analog of the YMH-model Bogomolny equations and constitute our main tool in constructing 1-soliton solutions of the dyonic models (1.1) and (1.3) in Sects. 4 and 5. Compared to the other methods of construction of soliton solutions as "vertex operators" (or $\tau$-functions, etc ) [17], [18, [19] they have the advantage in providing a simple proof of the topological character of the soliton's energy, momenta and action $S_{1-s o l}\left(A_{n}^{(1)}\right)$. It is shown in Sect. 3 that all conserved quantities $\left(E^{a x}, P^{a x}, M^{a x}, Q_{e l}^{a x}, Q_{\theta}^{a x}, Q_{k}^{t o p}, s^{a x}\right)$ characterizing $U(1)$ charged topological 1-solitons depend only on the asymptotics of the fields at $x \rightarrow \pm \infty$. Therefore their values can be calculated without the knowledge of the explicit form of the solutions.

The main result of this paper is the explicit construction of the charged topological 1-solitons of the axial and vector models (1.1) and (1.3) presented in Sect. 4 and 5. Their semiclassical spectrum (derived in Sect. 3,6 and 7) is shown to have the following promissing dyonic 3 form

$$
\begin{gathered}
Q_{e l}^{a x}=\beta_{0}^{2}\left(j_{e l}+\frac{\nu}{2 \pi} j_{\varphi}\right), \quad Q_{\theta}^{a x}=\frac{2 \pi \nu \gamma_{0}}{\nu-\frac{8 \pi}{\beta_{0}^{2}}} j_{\varphi}, \quad Q_{m a g}=\frac{4 \pi}{\beta_{0}^{2}} j_{\varphi}, \quad j_{\varphi}=0, \pm 1, \cdots, \pm(n-1) \\
M_{j_{e l}, j_{\varphi}}^{a x}=\frac{4 m n}{\beta_{0}^{2}}\left|\sin \frac{Q_{e l}^{a x}-\beta_{0}^{2} Q_{m a g}}{4 n}\right|, \quad s^{a x}=\frac{(n-1)}{2 \beta_{0}^{2}}\left(Q_{e l}^{a x}+\beta_{0}^{2} Q_{m a g}\right), \quad j_{e l}=0, \pm 1, \cdots
\end{gathered}
$$

( $\nu$ and $\gamma_{0}$ are arbitrary real numbers $; \beta=i \beta_{0}$ ) for the "axial" model (1.1) and a similar form with $\left(Q_{e l}^{a x}, Q_{\theta}^{a x}\right) \longrightarrow\left(Q_{\tilde{\theta}}^{v e c}, Q_{e l}^{v e c}\right)$ interchanged for its T-dual "vector" model (1.3). It is worthwhile to mention that $j_{\varphi}$ (and $\nu, \gamma_{0}$ )-dependent shifts of $Q_{e l}^{a x}$ and $Q_{\theta}^{a x}$ come from the topological $\theta$-terms $(2.28)$ and $(2.33)$ added to $\mathcal{L}_{a}$ and $\mathcal{L}_{v}$ respectively. Our proof that the "axial" and "vector" models 1-solitons have equal masses, energies and spins (see Sect. 5) represents an important step in testing the "off-critical" (i.e. nonconformal ) T-duality. An interesting property of the mass spectrum (1.5) is that its large $n$ limit

$$
M^{a x}(n \rightarrow \infty)=\frac{m}{\beta_{0}^{2}}\left|Q_{e l}^{a x}-\beta_{0}^{2} Q_{m a g}\right|
$$

coincides with the BPS bound for the masses of particular $\left(Q_{e l}^{(2)}=0=Q_{\text {mag }}^{(1)}\right)$ dyons of 4-D $N=2$ super YM theory, i.e. $M^{a x}(n \rightarrow \infty) \sim Z_{-}=Q_{e l}^{(1)}-Q_{m}^{(2)}$, 21.

Sect. 7 contains preliminary results concerning the exact quantization of the $A_{n}^{(1)}$ dyonic models in consideration. Our starting point is the path integral formulation of the models

\footnotetext{
3 to be compared with the semiclassical dyonic spectrum of YMH model (see for example sect.VI of ref. 200.
} 
(1.1) and (1.3) as gauged $\hat{H}_{-} \backslash A_{n}^{(1)} / \hat{H}_{+}$two loop WZW model (see refs. 22 ). We next derive the exact effective action for the $A_{n}^{(1)}$ dyonic integrable models following the method 23] developed for the corresponding conformal $\sigma$-models (i.e. $m=0$ case). It turns out that the relevant counterterms as well as the finite renormalization of the coupling constants $\beta_{\text {renorm }}^{2}$ are identical to those of the conformal models (i.e. $V_{\text {def }}=0$ in eqn. (1.2)). One can further speculate that eqns. (1.5) with $\beta_{0}^{2} \rightarrow \beta_{0 \text {,renorm }}^{2}=\frac{2 \pi}{k-n-1}$ represent the exact quantum 1-soliton spectrum. Few hints in favour of such conjecture are given in sect. 7.3. The complete answer of the question about the exact quantization of the electrically charged topological solitons of the dyonic integrable models (1.1) and (1.3), as well as whether they can be described as representations of the affine quantum group $U_{q}\left(A_{n}^{(1)}\right)$ requires further investigations.

An important feature of all $A_{n}^{(1)}$ abelian affine Toda models $(n>1)$ is known to be that their action (as well as the Hamiltonian ) becomes complex for imaginary coupling constants. The corresponding soliton solutions are also complex functions but their energy and momentum are real [24]. The only exception is the Sine-Gordon model (i.e. $A_{1}^{(1)}$ ) whose action, Hamiltonian, solitons and breathers are all real. A similar phenomena takes place for the $A_{n}^{(1)}$ dyonic IM $(n>1)$. There exist only two exceptional cases with real action, Hamiltonian, etc. The first is the $A_{1}^{(1)}$ vector (or axial) model (i.e. all $\varphi_{i}=0$ ) which is known as the Complex Sine-Gordon (or Lund-Regge) model [10, [12]. However it is not of dyonic type since its solitons are either charged but nontopological or neutral topological in the T-dual picture. The $A_{2}^{(1)}$-vector model represents the unique member of the $A_{n}^{(1)}$ family of models (1.1) and (1.3) that has real actions, soliton solutions, etc. After an appropriate change of variables, $A=e^{i \beta_{0} \theta} \sinh \left(\beta_{0} r\right), \quad B=-e^{-i \beta_{0} \theta} \sinh \left(\beta_{0} r\right), \quad c=e^{i \beta_{0} \varphi}$ we end up with the following real $\mathcal{L}_{v}$

$$
\mathcal{L}_{v}=\partial \varphi \bar{\partial} \varphi+\partial r \bar{\partial} r+\tanh ^{2}\left(\beta_{0} r\right) \partial \theta \bar{\partial} \theta+\frac{2 m^{2}}{\beta_{0}^{2}} \sinh \left(\beta_{0} r\right) \cos \beta_{0}(\theta+2 \varphi)
$$

The properties of its soliton solutions can be extracted from eqns. (5.8).

It is important to note that the $U(1)$ charged topological solitons do not exhaust all the particle-like finite energy solutions of the IM's (1.1) and (1.3). The dyonic IM's in consideration admit also $d$ "species" $(d=1,2, \cdots n-1)$ of neutral 1 -solitons that turns out to coincide with the $A_{n-1}^{(1)}$-abelian affine Toda solitons [17], [25]. The complete list of solutions also includes three type of breathers representing all the possible bound states of the charged and neutral 1-soliton and anti-solitons (i.e. "dyon-dyon", "dyon -monopole" condensates, etc.). The explicit construction of all these solutions and the discussion of their spectrum are presented in our work 22].

Sect. 8 contains preliminary discussion of the S- and T- duality properties of the considered integrable models. 


\section{$2 \quad A_{n}^{(1)}$-Dyonic Model}

2.1 Zero curvature and graded structures. The integrability of the axial $A_{n}^{(1)}$-model is a consequence of the fact that its equations of motion

$$
\begin{aligned}
& \partial\left(\frac{\bar{\partial} \psi e^{-\beta \varphi_{1}}}{\Delta}\right)+\beta^{2}\left(\frac{n+1}{2 n}\right) \frac{\psi e^{-2 \beta \varphi_{1}} \partial \chi \bar{\partial} \psi}{\Delta^{2}}+m^{2} e^{\beta \varphi_{n-1}} \psi=0, \\
& \bar{\partial}\left(\frac{\partial \chi e^{-\beta \varphi_{1}}}{\Delta}\right)+\beta^{2}\left(\frac{n+1}{2 n}\right) \frac{\chi e^{-2 \beta \varphi_{1}} \partial \chi \bar{\partial} \psi}{\Delta^{2}}+m^{2} e^{\beta \varphi_{n-1}} \chi=0 \\
& \partial \bar{\partial} \varphi_{l}+\beta\left(\frac{n-l}{n}\right) \frac{\partial \chi \bar{\partial} \psi e^{-\beta \varphi_{1}}}{\Delta^{2}}-\frac{m^{2}}{\beta} e^{\beta\left(\varphi_{l+1}+\varphi_{l-1}-2 \varphi_{l}\right)}+ \\
& +\frac{m^{2}}{\beta}\left(e^{\beta\left(\varphi_{1}+\varphi_{n-1}\right)}+\beta^{2} \frac{l}{n} \psi \chi e^{\beta \varphi_{n-1}}\right)=0,
\end{aligned}
$$

$l=1, \cdots, n-1$, can be written as zero curvature condition

$$
[\partial+\mathcal{A}, \bar{\partial}+\overline{\mathcal{A}}]=0 .
$$

The Lax connections $\mathcal{A}$ and $\overline{\mathcal{A}}$ (i.e. $\mathcal{A}_{\mu}$ ) lies in the $A_{n}^{(1)}$ centerless affine Kac-Moody algebra (loop algebra) t t and are given by

$$
\begin{aligned}
\mathcal{A} & =\beta^{2} \frac{\psi \partial \chi}{2 \Delta} e^{-\beta \varphi_{1}} \frac{\lambda_{1} \cdot H^{(0)}}{\lambda_{1}^{2}}+\frac{\beta}{2} \sum_{j=1}^{n-1} \partial \varphi_{j} h_{j+1}^{(0)}+\beta \frac{\partial \chi}{\Delta} e^{-\frac{1}{2} \beta \varphi_{1}} E_{-\alpha_{1}}^{(0)} \\
& +m\left(\sum_{j=1}^{n-1} e^{-\frac{1}{2} \beta\left(2 \varphi_{j}-\varphi_{j-1}-\varphi_{j+1}\right)} E_{-\alpha_{j+1}}^{(0)}+\beta \psi e^{\frac{1}{2} \beta \varphi_{n-1}} E_{\alpha_{1}+\alpha_{2}+\cdots \alpha_{n}}^{(-1)}+e^{\frac{1}{2} \beta\left(\varphi_{1}+\varphi_{n-1}\right)} E_{\alpha_{2}+\cdots \alpha_{n}}^{(-1)}\right) \\
\overline{\mathcal{A}} & =-\beta^{2} \frac{\chi \bar{\partial} \psi}{2 \Delta} e^{-\beta \varphi_{1}} \frac{\lambda_{1} \cdot H^{(0)}}{\lambda_{1}^{2}}-\frac{\beta}{2} \sum_{j=1}^{n-1} \bar{\partial} \varphi_{j} h_{j+1}^{(0)}-\beta \frac{\bar{\partial} \psi}{\Delta} e^{-\frac{1}{2} \beta \varphi_{1}} E_{\alpha_{1}}^{(0)} \\
& -m\left(\sum_{j=1}^{n-1} e^{-\frac{1}{2} \beta\left(2 \varphi_{j}-\varphi_{j-1}-\varphi_{j+1}\right)} E_{\alpha_{j+1}}^{(0)}+\beta \chi e^{\frac{1}{2} \beta \varphi_{n-1}} E_{-\left(\alpha_{1}+\alpha_{2}+\cdots \alpha_{n}\right)}^{(1)}+e^{\frac{1}{2} \beta\left(\varphi_{1}+\varphi_{n-1}\right)} E_{-\left(\alpha_{2}+\cdots \alpha_{n}\right)}^{(1)}\right)
\end{aligned}
$$

In the above particular choice for the connection $\mathcal{A}_{\mu}$ is hidden an interesting and rich algebraic structure known as the Leznov-Saveliev method [3] for constructing 2-D integrable models. In order to give an idea of how it works in our case of the singular non-abelian affine Toda (dyonic) models, we transform $\mathcal{A}$ and $\overline{\mathcal{A}}$ by an appropriate gauge transformation (dressing)

$$
\mathcal{A}_{\mu}^{W}=W \mathcal{A}_{\mu} W^{-1}+W \partial_{\mu} W^{-1}, \quad W^{-1}=e^{\frac{1}{2} \sum_{i=1}^{n-1} \beta \varphi_{i} h_{i+1}^{(0)}} e^{\beta \psi E_{\alpha_{1}}^{(0)}} e^{\frac{1}{2} \beta R \frac{\lambda_{1} \cdot H}{\lambda_{1}^{2}}}
$$

that leaves eqs. (2.1) unchanged and transforms the Lax connection into the following suggestive form

$$
\mathcal{A}^{W}=D^{-1} \partial D+\epsilon_{-}, \quad \overline{\mathcal{A}}^{W}=-D^{-1} \epsilon_{+} D
$$

\footnotetext{
4 all the algebraic definitions concerning $A_{n}^{(1)}$ are as in refs. 17, 22]
} 
where

$$
\epsilon_{ \pm}=m\left(\sum_{l=2}^{n} E_{ \pm \alpha_{l}}^{(0)}+E_{\mp\left(\alpha_{2}+\cdots+\alpha_{n}\right)}^{( \pm 1)}\right)
$$

The group element $D \in S L(2) \otimes U(1)^{(n-1)}$ is parametrized as follows

$$
\begin{aligned}
& D_{a}=e^{\frac{\beta R}{2 \lambda_{1}^{2}} \lambda_{1} \cdot H^{(0)}} e^{\beta \chi E_{-\alpha_{1}}^{(0)}} e^{\sum_{l=1}^{n-1} \beta \varphi_{l} h_{l+1}^{(0)}} e^{\beta \psi E_{\alpha_{1}}^{(0)}} e^{\frac{\beta R}{2 \lambda_{1}^{2}} \lambda_{1} \cdot H^{(0)}} \\
& =e^{\beta \tilde{\chi} E_{-\alpha_{1}}^{(0)}} e^{\frac{\beta R}{\lambda_{1}^{2}} \lambda_{1} \cdot H^{(0)}+\sum_{l=1}^{n-1} \beta \varphi_{l} h_{l+1}^{(0)}} e^{\beta \tilde{\psi} E_{\alpha_{1}}^{(0)}}
\end{aligned}
$$

As explained in refs. [14], [22] the $\epsilon_{ \pm}$-invariant subgroup $g_{0}^{0} \in U(1),\left[\epsilon_{ \pm}, g_{0}^{0}\right]=0$, is spanned by $\lambda_{1} \cdot H^{(0)}$, i.e. $g_{0}^{0}=e^{\frac{R}{\lambda_{1}^{2}} \lambda_{1} \cdot H^{(0)}}$, where $\lambda_{l}, l=1, \cdots n$ are the fundamental weights of $A_{n}$, $\lambda_{1}^{2}=\frac{n}{n+1}$. The appearence of the nonlocal field $R$ defined by

$$
\partial R=\beta \frac{\psi \partial \chi}{\Delta} e^{-\beta \varphi_{1}}, \quad \bar{\partial} R=\beta \frac{\chi \bar{\partial} \psi}{\Delta} e^{-\beta \varphi_{1}}
$$

is a consequence of the subsidiary constraint

$$
J=\operatorname{Tr}\left(D^{-1} \partial D \lambda_{1} \cdot H^{(0)}\right)=0, \quad \bar{J}=\operatorname{Tr}\left(\bar{\partial} D D^{-1} \lambda_{1} \cdot H^{(0)}\right)=0
$$

imposed on the zero grade conserved currents $(\partial \bar{J}=\bar{\partial} J=0)$ of the original two loop gauged $H_{-} \backslash A_{n}^{(1)} \otimes A_{n}^{(1)} / H_{+}$WZW model[22], [26]. We remind the algebraic recipe for constructing generic integrable models(see for example [17], [18, [19], 22]): Given an (finite or infinite dimensional ) algebra $\mathcal{G}_{n}^{(a)}$. Introduce a graded structure $\left(\mathcal{G}_{n}^{(a)}, Q\right)$ by means of the grading operator $Q$,

$$
\left[Q, \mathcal{G}_{l}\right]=l \mathcal{G}_{l}, \quad \mathcal{G}_{n}^{(a)}=\bigoplus \mathcal{G}_{l}, \quad\left[\mathcal{G}_{l}, \mathcal{G}_{k}\right] \in \mathcal{G}_{l+k} \quad l, k=0, \pm 1, \cdots
$$

A family of grade one integrable models $\left\{\mathcal{G}_{n}^{(a)}, Q, \epsilon_{ \pm}, g_{0}^{f} \subset \mathcal{G}_{0} / \mathcal{G}_{0}^{0}\right\}$ is defined by:

- an appropriate choice of grade one constant elements $\epsilon_{ \pm} \in \mathcal{G}_{ \pm 1}$.

- The 0-grade group element $D=\exp \left(\mathcal{G}_{0}\right)$ contains all fields $R(z, \bar{z}), \varphi_{l}(z, \bar{z}), \psi(z, \bar{z}), \chi(z, \bar{z})$, etc. appearing in (1.1), (2.1), (2.3).

- When $\mathcal{G}_{0}$ has an invariant subspace $\mathcal{G}_{0}^{0} \subset \mathcal{G}_{0}$, such that $\left[\epsilon_{ \pm}, \mathcal{G}_{0}^{0}\right]=0$, one can consider the subfamily of "singular" integrable models by imposing the subsidiary constraints

$$
\operatorname{Tr}\left(D^{-1} \partial D \mathcal{G}_{0}^{0}\right)=\operatorname{Tr}\left(\bar{\partial} D D^{-1} \mathcal{G}_{0}^{0}\right)=0
$$

allowing to eliminate the degree of freedom associated to $\mathcal{G}_{0}^{0}$.

- Finaly, with $\epsilon_{ \pm}, D=\exp \left(\mathcal{G}_{0}\right)$ and the subsidiary condition (2.8) we can construct the desired Lax connections $\mathcal{A}, \overline{\mathcal{A}}$ according to eqs. (2.4). 
The answer to the questions concerning the derivation of this recipe, its equivalence to the Hamiltonian reduction (Drinfeld-Sokolov) procedure and the two loop gauged $H_{-} \backslash G_{n}^{(a)} \otimes$ $G_{n}^{(a)} / H_{+}$WZW models as well as the classification of the grade one integrable models can be found in refs. [22]. The $A_{n}^{(1)}$-dyonic model (1.1) in consideration corresponds to the following specific choice of the grading operator

$$
Q=n \hat{d}+\sum_{l=2}^{n} \lambda_{l} \cdot H^{(0)}, \quad\left[\hat{d}, E_{\alpha}^{(p)}\right]=p E_{\alpha}^{(p)}, \quad\left[\hat{d}, h_{i}^{(p)}\right]=p h_{i}^{(p)}, \quad p=0, \pm 1, \cdots
$$

The grade one constant generators $\epsilon_{ \pm}$are those given in eq. (2.5) and $\mathcal{G}_{0}^{0}=\lambda_{1} \cdot H^{(0)}$.

2.2 $A_{n}^{(1)}$ - Vector Model. As we have mentioned, there exist two inequivalent ways (axial and vector) of gauge fixing the local $U(1)$ symmetry generated by the currents $\left(J_{\lambda_{1} \cdot H}, \bar{J}_{\lambda_{1} \cdot H}\right) \in$ $\mathcal{G}_{0}^{0}$. The details concerning the derivation of the axial and vector Lagrangeans, (1.1) and (1.3) from the ungauged one (1.4) are presented in our recent paper [15]. The problem we address here is about the nonlocal change of the axial variables $\psi, \chi, \varphi_{i}$ into the vector ones $A, B, c_{i}$. Observe that both, the axial model eqns. (2.1) as well as the vector model equations of motion

$$
\begin{aligned}
\partial \bar{\partial} c_{1} & =m^{2}\left(\frac{c_{2}}{c_{1}}-A c_{1}^{2} c_{2} \cdots c_{n-1}\right), \quad \partial \bar{\partial} c_{n-1}=m^{2}\left(\frac{B}{c_{1} \cdots c_{n-1}^{2}}-\frac{c_{n-1}}{c_{n-2}}\right), \\
\partial \bar{\partial} c_{k} & =m^{2}\left(\frac{c_{k+1}}{c_{k}}-\frac{c_{k}}{c_{k-1}}\right), \quad k=2,3, \cdots n-2 \\
\partial\left(\frac{\bar{\partial} A}{1-A B}\right) & =\frac{A \bar{\partial} A \partial B}{(1-A B)^{2}}+\frac{m^{2}}{c_{1} \cdots c_{n-1}^{2}} \\
\bar{\partial}\left(\frac{\partial B}{1-A B}\right) & =\frac{B \bar{\partial} A \partial B}{(1-A B)^{2}}+m^{2} c_{1}^{2} c_{2} \cdots c_{n-1}
\end{aligned}
$$

can be written in a compact form

$$
\bar{\partial}\left(D^{-1} \partial D\right)+\left[\epsilon_{-}, D^{-1} \epsilon_{+} D\right]=0, \quad \partial\left(\bar{\partial} D D^{-1}\right)-\left[\epsilon_{+}, D \epsilon_{-} D^{-1}\right]=0
$$

For the axial case we take $D=D_{a} \in g_{0}$ in the form (2.6), or equivalently in the following matrix representation

$$
\begin{gathered}
D_{a}=\left(\begin{array}{cc}
\tilde{d}_{2} & 0 \\
0 & \tilde{d}_{n-1}
\end{array}\right), \quad \tilde{d}_{n-1}=\operatorname{diag}\left(e^{\beta\left(\varphi_{2}-\varphi_{1}-\frac{R}{n}\right)}, \cdots, e^{-\beta\left(\varphi_{n-1}+\frac{R}{n}\right)}\right) \\
\tilde{d}_{2}=\left(\begin{array}{cc}
e^{\beta R} & \beta \psi e^{\beta \frac{n-1}{2 n} R} \\
\beta \chi e^{\beta \frac{n-1}{2 n} R} & e^{\beta\left(\varphi_{1}-\frac{R}{n}\right)}\left(1+\beta^{2} \psi \chi e^{-\beta \varphi_{1}}\right)
\end{array}\right)
\end{gathered}
$$

Eliminating further the field $R$ according to eqns. (2.7) we derive eqns. (2.1) from (2.10) and (2.7). The parametrization of $D \in S U(2) \otimes U(1)^{n-1}$ appropriate for the vector case is

$$
D_{v}=\left(\begin{array}{cc}
d_{2} & 0 \\
0 & d_{n-1}
\end{array}\right), \quad d_{n-1}=\operatorname{diag}\left(c_{1}, \cdots c_{n-1}\right)
$$




$$
d_{2}=\left(\begin{array}{cc}
A & u \\
\frac{A B-1}{u c_{1} c_{2} \cdots c_{n-1}} & \frac{B}{c_{1} c_{2} \cdots c_{n-1}}
\end{array}\right)
$$

where the nonlocal field $u$ ( the vector model analog of $R$ ) is defined by the following first order equations

$$
\partial \ln \left(u c_{1} \cdots c_{n-1}\right)=-\frac{A \partial B}{1-A B}, \quad \bar{\partial} \ln u=-\frac{B \bar{\partial} A}{1-A B}
$$

Starting from eqns. (2.10) with $D=D_{v}$ and taking into account (2.13) the result now is the vector model equations (2.9). It is then clear that comparing axial and vector parametrizations of $D$, i.e. $D_{a}=D_{v}$ we arrive at the desired axial-vector change of variables

$$
\begin{aligned}
e^{\beta R} & =A, \quad e^{-\beta \varphi_{k}}=c_{k} c_{k+1} \cdots c_{n-1} A^{\frac{n-k}{n}}, \quad k=1, \cdots, n-1 \\
\beta \psi & =u A^{-\frac{n-1}{2 n}}, \quad \beta \chi=\frac{A B-1}{u c_{1} c_{2} \cdots c_{n-1}} A^{-\frac{n-1}{2 n}},
\end{aligned}
$$

Equivalently the reverse vector-axial transformations are given by

$$
\begin{gathered}
c_{k}=e^{\beta\left(\varphi_{k+1}-\varphi_{k}-\frac{R}{n}\right)}, \quad A=e^{\beta R}, \quad B=e^{-\beta R}\left(1+\beta^{2} \psi \chi e^{-\beta \varphi_{1}}\right) \\
\tilde{u}^{2}=\frac{\psi}{\chi}, \quad \tilde{u}=u\left(\frac{c_{1} c_{2} \cdots c_{n-1}}{A B-1}\right)^{\frac{1}{2}}
\end{gathered}
$$

One can easely check that inserting (2.14) in eqns. (2.1) gives the vector model equations (2.9). It is worthwhile to mention that axial gauge fixing corresponds to the elimination of the field $R$ from $\mathcal{G}_{0}^{0}$ constraints, $J_{\lambda_{1} \cdot H}=\bar{J}_{\lambda_{1} \cdot H}=0$, i.e. eqns. (2.7) in this case. In this language, the vector gauge fixing is equivalent to the elimination of another field $\frac{\psi}{\chi}=\tilde{u}^{2}$ from the same constraint equations.

Performing the above change of variables in $\mathcal{L}_{v}$ we find the following relation including the so called generating function 15$] \mathcal{F}$ :

$$
\mathcal{L}_{v}=\mathcal{L}_{a}+\frac{d \mathcal{F}}{d t}, \quad \frac{d \mathcal{F}}{d t}=\frac{1}{2 \beta}\left(\partial R \bar{\partial} \ln \left(\frac{\chi}{\psi}\right)-\bar{\partial} R \partial \ln \left(\frac{\chi}{\psi}\right)\right)
$$

It is not surprising that the same relation (2.16) appears as a result of abelian T-duality transformation between the axial and vector IM's in consideration (see Sect 3 of ref. [15]). Denote by $\theta=-\frac{i}{2 \beta_{0}} \ln \left(\frac{\chi}{\psi}\right)$ (for the axial model (1.1)) and $\tilde{\theta}=\frac{2}{i \beta_{0}} \ln A=2 R$ (for the vector model ) the corresponding isometric coordinates $\emptyset$ and by $\Pi_{\theta}$ and $\Pi_{\tilde{\theta}}$ their conjugate momenta. As it is well known [15], 28] the following canonical transformation

$$
\Pi_{\tilde{\theta}}=-\partial_{x} \theta, \quad \Pi_{\theta}=-\partial_{x} \tilde{\theta}
$$

(and all remaining $\Pi_{\varphi_{i}}, \varphi_{i}$ unchanged) acts as T-duality transformation with generating function $\mathcal{F}$ 28]. Then, the relation $(2.16)$ between vector and axial Lagrangeans is a simple consequence of the fact that both Hamiltonians are equal, i.e. $H_{a}=H_{v}$. Therefore the

\footnotetext{
${ }^{5} \theta \rightarrow \theta+a$ and $\tilde{\theta} \rightarrow \tilde{\theta}+\tilde{a}$ are symmetries of $\mathcal{L}_{a}$ and $\mathcal{L}_{v}$, that coincides with their global $U(1)$ symmetry
} 
nonlocal change of variables (2.14) represent an integrated form of the T-duality transformations (2.17) accompanied by certain point transformations of the rest of the (nonisometric ) variables $\varphi_{k} \rightarrow c_{k}=f_{k}\left(\varphi_{i}, R\right)$.

2.3 Symmetries and Vacua. It is important to note that both, the Noether symmetries of eqs. (2.1) ( and $\mathcal{L}_{a}$ in (1.1)), as well as the multiple zeros of the potential (1.1)

$$
V_{a}=\frac{1}{\beta^{2}}\left(\operatorname{Tr}\left(\epsilon_{+} D \epsilon_{-} D^{-1}\right)-m^{2} n\right)
$$

are encoded in the algebraic data: $\left(Q, \epsilon_{ \pm}, \mathcal{G}_{0}^{0}\right)$. Writing the field equations (2.1) in a compact form (2.10) and taking traces with $h_{0}^{i}$, we verify that all the elements $h_{0}^{i}$ of $\mathcal{G}_{0}^{0} \subset \mathcal{G}_{0}\left(\left[h_{0}^{i}, \epsilon_{ \pm}\right]=\right.$ $\left.0, \quad i=1, \cdots, K_{0}=\operatorname{dim} \mathcal{G}_{0}^{0}\right)$ generate continuous symmetries of eqn. (2.10)

$$
D^{\prime}=e^{-i \beta_{0}^{2} \sum_{l=1}^{K_{0}} a^{l} h_{0}^{l}} D
$$

In our case $K_{0}=1, h_{0}^{(1)}=\lambda_{1} \cdot H^{(0)}$ and the above transformation is a global $U(1)$ (electric) symmetry of (1.1) mentioned in Sect. 1. For imaginary coupling $\beta=i \beta_{0}$, the $A_{n}^{(1)}$-dyonic Lagrangean (1.1) ( as well as the potential (2.18) ) are invariant under the following discrete group transformations

$$
\varphi_{l}^{\prime}=\varphi_{l}+\frac{2 \pi}{\beta_{0}} \frac{l}{n} N, \quad l=1, \cdots, n-1, \quad \psi^{\prime}=e^{i \pi\left(\frac{N}{n}+s_{1}\right)} \psi, \quad \chi^{\prime}=e^{i \pi\left(\frac{N}{n}+s_{2}\right)} \chi
$$

for $N$ an arbitrary integer and $s_{a}, a=1,2$ are both even ( odd) integers ( i.e., $s_{1}+s_{2}=$ $\left.2 S^{\prime}, s_{1}-s_{2}=2 L^{\prime}\right)$. It is also invariant under CP transformations $(P: x \rightarrow-x)$

$$
\varphi_{l}^{\prime \prime}=\varphi_{l}, \quad \psi^{\prime \prime}=\chi, \quad \chi^{\prime \prime}=\psi
$$

It is convenient to parametrize $\psi$ and $\chi$ as

$$
\psi=\frac{1}{\beta_{0}} e^{i \beta_{0}\left(\frac{1}{2} \varphi_{1}-\theta\right)} \sqrt{\frac{2 n}{n+1}} \sinh \left(\beta_{0} r\right), \quad \chi=\frac{1}{\beta_{0}} e^{i \beta_{0}\left(\frac{1}{2} \varphi_{1}+\theta\right)} \sqrt{\frac{2 n}{n+1}} \sinh \left(\beta_{0} r\right)
$$

in terms of one noncompact $(r)$ and two compact $\left(\varphi_{1}, \theta\right)$ fields following the tradition of the 3 -D black string constructions [0. The $\psi, \chi$ discrete transformations (2.20) and (2.21) then take the form

$$
\begin{aligned}
& r^{\prime}=r, \quad \theta^{\prime}=\theta+\frac{\pi}{\beta_{0}} L^{\prime}, \\
& r^{\prime \prime}=r, \quad \theta^{\prime \prime}=-\theta+\frac{2 \pi}{\beta_{0}} L^{\prime \prime}
\end{aligned}
$$

with $L^{\prime}$ and $L^{\prime \prime}$ arbitrary integers. As a consequence of (2.20) and (2.21) our dyonic model (1.1) possesses together with the trivial classical vacuum solution $D=1,\left(N=0, \varphi_{l}=\right.$ $0, \psi \chi=0, \ln \frac{\psi}{\chi}=$ const., $\left.V(D=1)=0\right)$ an infinite set of new distinct vacua

$$
\varphi_{l}^{(N)}=\frac{2 \pi}{\beta_{0}} \frac{l}{n} N, \quad r^{(0)}=0, \quad \theta^{(L)}=-\frac{i}{2 \beta_{0}} \ln \left(\frac{\chi^{(L)}}{\psi^{(L)}}\right)=\frac{\pi L}{2 \beta_{0}}, \quad L=2 L^{\prime \prime}-L^{\prime}
$$

\footnotetext{
${ }^{6}$ our $n=2$ "free" $\mathcal{L}$ (i.e. $V=0$ ) after an appropriate field redefinitions (reflecting another $U(1)$ gauge fixing ) and with the counterterms (7.9) and (7.10) included coincides with the euclidean 3-D black string of ref. [27].
} 
This provides a new set of allowed boundary conditions for the fields at $x \rightarrow \pm \infty$ :

$$
\varphi_{l}^{(N)}( \pm \infty)=\frac{2 \pi}{\beta_{0}} \frac{l N_{ \pm}}{n}, \quad r( \pm \infty)=0, \quad \theta^{(L)}( \pm \infty)=\frac{\pi L_{ \pm}}{2 \beta_{0}}
$$

(i.e. $\psi( \pm \infty)=\chi( \pm \infty)=0$, but $\frac{\psi}{\chi}( \pm \infty) \neq 0$ ). The multiply degenerate vacuum of our axial integrable model (1.1) is an indication that it admits finite energy topological solitons solutions interpolating between two different vacua $\left(N_{ \pm}, L_{ \pm}\right): j_{\varphi}=N_{+}-N_{-}(\bmod n), j_{\theta}=$ $L_{+}-L_{-}$. Due to the $U(1)$ symmetry (2.19) $\left(K_{0}=1\right)$ such solutions might also carry nontrivial electric charge:

$$
Q_{e l}^{a x}=\int_{-\infty}^{\infty} d x J_{e l}^{0, a x}=2 \beta_{0} \int_{-\infty}^{\infty} d x \partial_{x} R, \quad J_{e l}^{\mu, a x}=2 \beta_{0} \epsilon^{\mu \nu} \partial_{\nu} R
$$

where $R$ is defined in (2.7). The topological current

$$
J_{\theta}^{\mu, a x}=\beta_{0} \epsilon^{\mu \nu} \partial_{\nu} \theta\left(=\frac{1}{2 i} \epsilon^{\mu \nu} \partial_{\nu} \ln \left(\frac{\chi}{\psi}\right)\right), \quad Q_{\theta}^{a x}=\frac{\pi j_{\theta}}{2}, \quad j_{\theta}=0, \pm 1, \cdots
$$

appears to be T-dual to the electric current $J_{e l}^{\mu, v e c}$ (see Sect. 2.5 and ref. [15]). It plays the role of electric current in the T-dual model (1.3) that generates the global $U(1)$ transformation: $A^{\prime}=e^{i a \beta_{0}^{2}} A, B^{\prime}=e^{-i a \beta_{0}^{2}} B, c_{k}^{\prime}=e^{-i \frac{a}{n} \beta_{0}^{2}} c_{k}$. Similarly to the free case, $(V=0)$, 28] the currents $J_{e l}^{\mu, a x}, J_{\theta}^{\mu, a x}$ ( and their conjugate "coordinates" $\theta$ and $2 R \equiv \tilde{\theta}$ ) form a canonical pairs

$$
\left\{\frac{1}{\beta_{0}} J_{e l}^{0, a x}\left(x, t_{0}\right), \theta\left(y, t_{0}\right)\right\}=-\left\{\tilde{\theta}\left(x, t_{0}\right), \frac{1}{\beta_{0}} J_{\theta}^{0, a x}\left(y, t_{0}\right)\right\}=\delta(x-y)
$$

but $\left\{Q_{e l}^{a x}, Q_{\theta}^{a x}\right\}=0$. Note that the other topological currents

$$
J_{k}^{\mu}=\frac{2 n}{\beta_{0}} \epsilon^{\mu \nu} \partial_{\nu} \varphi_{k}, \quad Q_{k}^{t o p}=\int_{-\infty}^{\infty} J_{k}^{0} d x=k Q_{m a g}, \quad Q_{m a g}=\frac{4 \pi}{\beta_{0}^{2}} j_{\varphi}
$$

( $Q_{m a g}$ stands for the magnetic charge) are not T-duals to the corresponding currents $\tilde{J}_{k}^{\mu}$ (5.14) of the vector model (1.3).

The origin of the discrete symmetry (2.20) is in the following continuous symmetry of the $V=0$ free model $(1.1)$

$$
\begin{aligned}
& J_{\varphi_{1}}^{\mu}: \varphi_{1}^{\prime}=\varphi_{1}+a_{1}, \quad \psi^{\prime}=e^{\frac{i \beta_{0} a_{1}}{2}} \psi, \quad \chi^{\prime}=e^{\frac{i \beta_{0} a_{1}}{2}} \chi \\
& J_{\varphi_{k}}^{\mu}: \quad \varphi_{k}^{\prime}=\varphi_{k}+a_{k}, \quad \psi^{\prime}=\psi, \quad \chi^{\prime}=\chi, \quad k \neq 1 \\
& J_{e l}^{\mu}: \quad \varphi_{l}^{\prime}=\varphi_{l}, \quad \psi^{\prime}=e^{i a \beta_{0}^{2}} \psi, \quad \chi^{\prime}=e^{-i a \beta_{0}^{2}} \chi, \quad l=1,2, \cdot, n-1
\end{aligned}
$$

broken to $(2.23)$ when $V \neq 0$ is added. It is important to mention an interesting relation between the two $U(1)$ currents $J_{\varphi_{1}}^{\mu}, J_{e l}^{\mu}$ and the topological current $I_{\varphi_{1}}^{\mu}=\epsilon^{\mu \nu} \partial_{\nu} \varphi_{1}$ which, say for $n=2$,reads

$$
J_{e l}^{\mu}=2 \epsilon^{\mu \nu} J_{\nu}^{\varphi_{1}}-2 I_{\varphi_{1}}^{\mu}
$$

2.4 Topological $\theta$-term. Although eqn. (2.27) takes place in the $V=0$ model only, the fact that the electric charge gets contributions from the topological charge $Q_{1}^{\text {top }}$ persists in the 
general case where $V \neq 0$ (1.1) as we shall show on the example of the 1-soliton solutions in Sect. 3. As one can expect the dyonic properties of our model (1.1) are consequences of the CPT breaking term $\frac{\epsilon^{\mu \nu} \partial_{\mu} \psi \partial_{\nu} \chi}{\Delta} e^{-i \beta_{0} \varphi_{1}}=\delta \mathcal{L}_{C P T}$ which, by the following change of variables, $\psi_{0}=e^{-i \frac{\beta_{0}}{2} \varphi_{1}} \psi, \chi_{0}=e^{-i \frac{\beta_{0}}{2} \varphi_{1}} \chi$ reads,

$$
\delta \mathcal{L}_{C P T}=\frac{\epsilon^{\mu \nu}}{\Delta}\left\{\partial_{\mu} \psi_{0} \partial_{\nu} \chi_{0}-\frac{i n}{\beta_{0}(n+1)} \partial_{\mu} \varphi_{1} \partial_{\nu} \ln \left(\frac{\chi_{0}}{\psi_{0}}\right)\right\}+\frac{i n}{\beta_{0}(n+1)} \epsilon^{\mu \nu} \partial_{\mu} \varphi_{1} \partial_{\nu} \ln \left(\frac{\chi_{0}}{\psi_{0}}\right)
$$

The pure topological term

$$
\frac{i n}{\beta_{0}(n+1)} \partial^{\nu}\left(\epsilon_{\mu \nu} \ln \left(\frac{\chi_{0}}{\psi_{0}}\right) \partial^{\mu} \varphi_{1}\right) \equiv \frac{2 n}{n+1} \epsilon^{\mu \nu} \partial_{\mu} \varphi_{1} \partial_{\nu} \theta
$$

(being a total derivative) does not contribute to the equations of motion (2.1), but makes evident the top-charge contribution to the electric current (2.24), $\delta J_{e l}^{\mu}=\frac{2 n}{n+1} \beta_{0} \epsilon^{\mu \nu} \partial_{\nu} \varphi_{1}$. We further observe that the specific value of the coefficient multiplying the topological current is irrelevant since one can make it arbitrary by adding to the original Lagrangean (1.1) certain " $\theta$-type " topological terms 凹

$$
\delta \mathcal{L}_{\text {top }}^{a}=i \frac{\beta_{0}}{8 \pi^{2}} \sum_{k=1}^{n-1} \nu_{k} \epsilon^{\mu \nu} \partial_{\mu} \varphi_{k} \partial_{\nu} \ln \left(\frac{\chi}{\psi}\right)
$$

where $\nu_{k}$ are arbitrary real constants. As a result, the improved electric current ( calculated from $\mathcal{L}_{\text {impr }}=\mathcal{L}+\delta \mathcal{L}_{\text {top }}$ ) has the form

$$
J_{e l, i m p r}^{\mu, a x}=2 \beta_{0} \epsilon^{\mu \nu} \partial_{\nu} R-\frac{\beta_{0}^{3}}{4 \pi^{2}} \epsilon^{\mu \nu} \sum_{k=1}^{n-1} \nu_{k} \partial_{\nu} \varphi_{k}
$$

Therefore we find that $Q_{e l}^{a x}$ is shifted by $\nu j_{\varphi}$ :

$$
\int_{-\infty}^{\infty} J_{e l, i m p r}^{0, a x} d x=Q_{e l}^{a x}-\frac{\nu \beta_{0}^{2}}{2 \pi} j_{\varphi}, \quad \nu=\frac{1}{n} \sum_{k=1}^{n-1} k \nu_{k}, \quad j_{\varphi}=0, \pm 1, \cdots, \pm(n-1)
$$

Similar phenomenon takes place in the open string with one compactified dimension (say $X_{25} \equiv \theta=\frac{1}{2 \beta} \ln \frac{\psi}{\chi}$ ) and boundary (Chan-Paton) term included ( see Sect 8.6 of ref. [30]). The string momenta $P_{25}$ gets contribution from the boundary (Wilson line) term $\Delta S_{\text {string }}=$ $i \oint A_{25}\left(X_{i}\right) d X_{25}$. Such similarity is not occasional, since in our case the electric current $J_{e l}^{a x}$ coincides with the momenta $\Pi_{\theta}$ conjugate to the "field coordinate " $\theta$, which for 1-soliton solutions (4.9) turns out to be periodic. Then the $\theta$-term (2.28) we have added to the original action (1.1) can be rewritten as the Wilson line of certain background gauge field $A_{a}\left(X_{k}\right)\left(a=1,2, \cdots n+1\right.$ and $\left.X_{k}=\left\{\varphi_{i}, \theta, \ln \Delta\right\}, k=1,2, \cdots n+1\right)$ :

$$
\delta S_{\text {top }}^{a}=\int d^{2} z \delta \mathcal{L}_{\text {top }}^{a}=\left(\frac{\beta}{4 \pi}\right)^{2} \oint d t \nu_{i} \varphi_{i} \partial_{t} \theta=\oint d \theta A_{\theta}\left(X_{k}\right)
$$

\footnotetext{
${ }^{7}$ We restrict ourselves to consider $\delta \mathcal{L}_{\text {top }}^{a}$ in a particular form (2.28), although one can include more $\theta$-terms, say, $\nu_{i j} \partial_{\mu} \varphi_{i} \partial_{\nu} \varphi_{j} \epsilon^{\mu \nu}+\nu_{i 0} \epsilon^{\mu \nu} \partial_{\mu} \varphi_{i} \partial_{\nu} \ln \Delta+\cdots$. The only contribution to $Q_{e l}$ comes from (2.28).
} 
i.e. $A_{\theta}=\left(\frac{\beta}{4 \pi}\right)^{2} \sum_{i=1}^{n-1} \nu_{i} \varphi_{i}$ and $A_{a}=0$ for $a \neq \theta$. Therefore our specific $U(1)^{n-1}$ linear gauge potential $A_{a}(X)$ corresponds to constant " electromagnetic field " $F_{a b}=\partial_{a} A_{b}-\partial_{b} A_{a}$, i.e. $F_{\theta i}=\left(\frac{\beta}{4 \pi}\right)^{2} \nu_{i}$ and all the other components vanish.

2.5 Axial vs. Vector $\theta$-terms. The shift of the electric charge $Q_{e l}^{a x} \rightarrow Q_{e l}^{a x}-\frac{\nu \beta_{0}^{2}}{2 \pi} j_{\varphi}$ induced by the topological term (2.28) together with the fact that $Q_{\theta}^{a x}$ is equal to the vector model electric charge $Q_{e l}^{v}$ (i.e. $Q_{e l}^{a x}, Q_{\theta}^{a x} \rightarrow Q_{\tilde{\theta}}^{v}, Q_{e l}^{v}$ by T-duality) addresses the question about the changes in $Q_{\theta}^{a x}$ caused by (2.28). Its answer requires the explicit form of the vector model $\theta$-term, that corresponds to the axial one $(2.28)$ via T-duality transformation. Applying the axial-vector change of variables (2.14) in eqn. (2.28) results in a nonlocal $\theta$ term (including $\tilde{u}$ ) for the vector model. This is an indication that one should consider new canonical transformations (more general than (2.17)) in order to have local $\theta$-terms for the vector model. Therefore we choose

$$
\begin{aligned}
\tilde{\theta}_{i m p r} & =\tilde{\theta}+\sum_{k=1}^{n-1} \alpha_{k} \varphi_{k}, \quad \theta_{i m p r}=\theta+\sum_{k=1}^{n-1} a_{k} \varphi_{k}+a_{0} \ln \left(1+\beta^{2} \psi \chi e^{-\beta \varphi_{1}}\right) \\
\tilde{\theta} & =2 R, \quad \theta=\frac{1}{2 \beta} \ln \left(\frac{\chi}{\psi}\right)
\end{aligned}
$$

as new isometric coordinates. Next, we define the standard (T-duality) canonical transformation (2.17) in terms of the new isometric coordinates $\tilde{\theta}_{i m p r}, \theta_{i m p r}$ and their conjugate momenta (together with the point transformations in the first line of eqn. (2.15)). In order to simplify the calculation we seek for the vector $\theta$-term in a form:

$$
\delta \mathcal{L}_{\text {top }}^{v}=\sum_{k=1}^{n-1} \frac{\gamma_{k}}{\beta^{2}} \epsilon^{\mu \nu} \partial_{\mu} \ln \left(c_{k} \cdots c_{n-1}\right) \partial_{\nu} \ln \left(\frac{A}{B}\right)
$$

The consistency with the "improved" T-duality transformation (with $\tilde{\theta}_{i m p r}, \theta_{i m p r}$ ) determines the unknown constants $\alpha_{k}, a_{k}, a_{0}$ and $\gamma_{k}$ as follows:

$$
\begin{aligned}
\alpha_{k} & =\frac{\nu_{k} \beta^{2}}{4 \pi^{2}}, \quad a_{k}=-\gamma_{k}, \quad a_{0}=\frac{1}{2 n \beta} \sum_{k=1}^{n-1} \gamma_{k}(k-n) \\
\nu_{k} & =\frac{8 \pi^{2} n}{\beta^{2}} \frac{\gamma_{k}}{\sum_{k=1}^{n-1} \gamma_{k}(n-k)}, \quad \frac{\gamma_{k}}{\nu_{k}}=\frac{\gamma_{l}}{\nu_{l}}=-\frac{a_{0} \beta^{3}}{4 \pi^{2}}, \quad k, l=1,2, \cdots, n-1
\end{aligned}
$$

As it is well known the $U(1)$ - currents and the $\theta$ and $\tilde{\theta}$ topological currents of the axial and vector models are related as follows:

$$
J_{e l, i m p r}^{0, a x}=\beta_{0} \partial_{x} \tilde{\theta}_{i m p r}=J_{\tilde{\theta}}^{0, v e c} \quad J_{e l, i m p r}^{0, v e c}=\beta_{0} \partial_{x} \theta_{i m p r}=J_{\theta}^{0, a x}
$$

The corresponding charges calculated from the Lagrangeans with $\theta$-terms and by using the explicit form (2.32) of $\tilde{\theta}_{i m p r}\left(\right.$ and $\left.\theta_{i m p r}\right)$ indeed coincide,

$$
Q_{e l, i m p r}^{a x}=Q_{\tilde{\theta}}^{v e c}=Q_{e l}^{a x}-\frac{\nu \beta_{0}^{2}}{2 \pi} j_{\varphi}, \quad Q_{e l, i m p r}^{v e c}=Q_{\theta}^{a x}=Q_{\theta}+2 \pi \tilde{\gamma} j_{\varphi}
$$


The new parameters $\tilde{\gamma}, \gamma_{0}, \nu_{0}$ and $\nu$ are defined by

$$
\tilde{\gamma}=\frac{1}{n} \sum_{k=1}^{n-1} k \gamma_{k}, \quad \gamma_{0}=\sum_{k=1}^{n-1} \gamma_{k}, \quad \nu=\frac{1}{n} \sum_{k=1}^{n-1} k \nu_{k}, \quad \nu_{0}=\sum_{k=1}^{n-1} \nu_{k}
$$

and satisfy the following relations

$$
\nu_{0}=\nu+\frac{8 \pi^{2}}{\beta^{2}}, \quad \nu=\frac{8 \pi^{2}}{\beta^{2}} \frac{\tilde{\gamma}}{\gamma_{0}-\tilde{\gamma}}, \quad \tilde{\gamma}=\frac{\gamma_{0} \nu}{\nu+\frac{8 \pi^{2}}{\beta^{2}}}
$$

Eqns. (2.37) are direct consequence of the above definitions and eqns. (2.34). Finally, the shifts in the charges due to the $\theta$-terms take the form:

$$
Q_{e l, i m p r}^{a x}=Q_{e l}^{a x}-\frac{\beta^{2} \nu}{2 \pi} j_{\varphi}, \quad Q_{\theta, i m p r}^{a x}=Q_{\theta}+\frac{2 \pi \gamma_{0} \nu}{\nu+\frac{8 \pi^{2}}{\beta^{2}}}, \quad Q_{\theta}=\frac{\pi}{2} j_{\theta}
$$

2.6 More discrete symmetries. The "translational type" $\left(\varphi_{l}, \theta\right)$-symmetries (2.20) that allows us to determine the vacua lattice $\left(\varphi_{l}^{(N)}, \theta^{(N)}\right)$ (2.23) does not exhaust all the discrete symmetries of the $A_{n}^{(1)}$ dyonic Lagrangean (1.1). By analogy with the $S_{n}$ symmetries of the $A_{n}$-abelian Toda models one expects certain $\left(\varphi_{l}, \theta\right)$ - "rotational" symmetries to take place. It is easy to verify that the vector model (1.3) is invariant under the following transformation

$$
A^{\prime}=B, \quad B^{\prime}=A, \quad c_{k}^{\prime}=\frac{1}{c_{n-k}}, \quad k=1,2, \cdots n-1
$$

The derivation of their counterpart that leaves invariant the axial gauged model (1.1) is however far from obvious. We start with the observation that the eqs. (2.10) as well as the potential (2.18) remain invariant $\left(V\left(D^{\prime}\right)=V(D)\right)$ under the transformation $\Pi: D^{\prime}=\Pi\left(D^{-1}\right)$ with the following properties:

$$
\Pi\left(\epsilon_{+}\right)=\epsilon_{-}, \quad \Pi\left(E_{\alpha_{1}}\right)=E_{\Pi\left(\alpha_{1}\right)}=E_{\alpha_{1}}, \quad \Pi^{2}=1
$$

combined with the space reflection $P: P \partial=\bar{\partial}, P^{2}=1$. The next step is to explicitly construct $\Pi$ with the above properties in terms of the elements $w_{k}=w_{\alpha_{k}},\left(w_{k} \beta=\beta-\left(\alpha_{k}\right.\right.$. $\beta) \alpha_{k}$ ) of the Weyl group of $A_{n}^{(1)}$. Define a composite transformation $(n \geq 3)$,

$$
\begin{aligned}
\Pi_{0}^{(n=2 j)} & =t_{j+1}^{j+2} t_{j}^{j+3} \cdots t_{4}^{2 j-1} t_{3}^{2 j}, \\
\Pi_{0}^{(n=2 j-1)} & =t_{j+1}^{j+1} t_{j}^{j+2} \cdots t_{4}^{2 j-2} t_{3}^{2 j-1}, \quad j=2,3, \cdots
\end{aligned}
$$

where $t_{k}^{l}=w_{l} w_{l-1} \cdots w_{k+1} w_{k} w_{k+1} \cdots w_{l-1} w_{l}, l \geq k, t_{k}^{k}=w_{k}$. One can prove by induction its main property, namely

$$
\Pi_{0}^{(n)}\left(\alpha_{1}\right)=\alpha_{1}, \quad \Pi_{0}^{(n)}\left(\alpha_{2}\right)=\alpha_{2}+\alpha_{3}+\cdots+\alpha_{n}, \quad \Pi_{0}^{(n)}\left(\alpha_{i}\right)=-\alpha_{n-i+3}, i=3,4, \cdots n
$$

Since $\Pi_{0}\left(E_{\beta}^{(k)}\right)=E_{\Pi_{0}(\beta)}^{(k)}$ we realize that

$$
\Pi_{0}\left(\epsilon_{+}\right)=m\left(E_{-\alpha_{2}}^{(1)}+E_{-\alpha_{3}}^{(0)}+\cdots+E_{-\alpha_{n-1}}^{(0)}+E_{-\alpha_{n}}^{(0)}+E_{\alpha_{2}+\alpha_{3} \cdots+\alpha_{n}}^{(0)}\right)
$$


Remember that $E_{\beta}^{(l)}$ can be represented as $E_{\beta}^{(l)}=\lambda^{l} \otimes E_{\beta}^{(0)}$ and $\hat{d}=\lambda \frac{d}{d \lambda}$. We next define an operator $T_{3}=\lambda_{2} \cdot H^{(0)}+2 \hat{d}$ such that

$$
\begin{gathered}
\Omega\left(E_{-\alpha_{2}}^{(1)}\right)=e^{-\ln (\lambda) T_{3}} E_{-\alpha_{2}}^{(1)} e^{\ln (\lambda) T_{3}}=\frac{1}{\lambda} E_{-\alpha_{2}}^{(1)}=E_{-\alpha_{2}}^{(0)} \\
\Omega\left(E_{\alpha_{2}+\cdots+\alpha_{n}}^{(0)}\right)=\frac{1}{\lambda} E_{\alpha_{2}+\cdots+\alpha_{n}}=E_{\alpha_{2}+\cdots+\alpha_{n}}^{(-1)}, \quad \Omega\left(E_{ \pm \alpha_{k}}^{(0)}\right)=E_{ \pm \alpha_{k}}^{(0)}, k=3,4, \cdots n
\end{gathered}
$$

Therefore

$$
e^{-\ln (\lambda) T_{3}} \Pi_{0}\left(\epsilon_{+}\right) e^{\ln (\lambda) T_{3}}=\epsilon_{-}
$$

and as a result we find that the operator $\Pi$ has the form $\Pi=\Omega \Pi_{0}$.

Taking into account the explicit parametrization (2.6) and (2.11) of $D_{a}$ we derive the following field transformations obtained from $D^{\prime}=\Pi\left(D^{-1}\right)$,

$$
\begin{aligned}
\varphi_{k}^{\prime} & =\varphi_{n-k+1}-\varphi_{1}-\frac{n-k}{\beta n} \ln \left(\Delta_{0}\right), \quad k=1,2, \cdots, n-1, \quad \varphi_{n}=0 \\
R^{\prime} & =-R+\frac{1}{\beta} \ln \Delta_{0}, \quad \Delta_{0}=1+\beta^{2} \psi \chi e^{-\beta \varphi_{1}} \\
\psi^{\prime} & =-\psi e^{-\beta \varphi_{1}} \Delta_{0}^{\frac{1-n}{2 n}}, \quad \chi^{\prime}=-\chi e^{-\beta \varphi_{1}} \Delta_{0}^{\frac{1-n}{2 n}}
\end{aligned}
$$

They leave invariant the potential: $V\left(D^{\prime}\right)=V(D)$ and combined with $P$ (i.e. $P \Pi$ ), generate symmetries of the action (1.1). The Lagrangean (1.1) transforms modulo total derivatives $\mathcal{L}\left(D^{\prime}\right)=\mathcal{L}(D)+\partial_{\mu} L^{\mu}$, (see Sect. 8 of ref. [14). We have to mention that (2.41) can be obtained from the vector model transformations (2.39) applying the axial-vector (non-local) change of variables (2.14). As we shall see in Sect. 3, the discrete symmetries (2.41) play a crucial role in the derivation of the first order "solitonic" equations (3.5).

2.7 Weyl families of IM's. The algebraic (Weyl group) constructions used in the discussion of the discrete symmetries of (1.1) addresses the question of whether the remaining Weyl group elements ( or their specific combinations including, say $w_{\alpha_{1}}$, etc. ) act as symmetries of our model (1.1) and if not, whether the Lagrangeans obtained represent new integrable models. This problem appears to be the nonconformal generalization of the Weyl families of conformal non-abelian Toda models constructed in ref. [14 ( see Sect. 8). The simplest transformation $D^{\prime}=w_{\alpha_{1}}(D)$ is not a symmetry of (1.1). It has the following components form $\left(D^{\prime}=\left(\psi_{0}, \chi_{0}, \varphi_{0 i}\right)\right)$

$$
\begin{aligned}
\varphi_{k} & =\varphi_{0 k}-\frac{n-k}{\beta n} \ln \left(1+\beta^{2} \psi_{0} \chi_{0} e^{\beta \varphi_{01}}\right) \\
\psi & =\chi_{0} e^{\beta \varphi_{01}}\left(1+\beta^{2} \psi_{0} \chi_{0} e^{\beta \varphi_{01}}\right)^{-\frac{n-1}{2 n}} \\
\chi & =\psi_{0} e^{\beta \varphi_{01}}\left(1+\beta^{2} \psi_{0} \chi_{0} e^{\beta \varphi_{01}}\right)^{-\frac{n-1}{2 n}}
\end{aligned}
$$

As a result of this change of variables the $w_{\alpha_{1}}$-image of (1.1) is an integrable model with Lagrangean $\mathcal{L}_{w_{\alpha_{1}}}$,

$$
\mathcal{L}_{w_{\alpha_{1}}}=\frac{1}{2} \eta_{i j} \partial \varphi_{0 i} \bar{\partial} \varphi_{0 j}+\frac{\partial \chi_{0} \bar{\partial} \psi_{0} e^{\beta \varphi_{01}}}{1+\beta^{2} \frac{n+1}{2 n} \psi_{0} \chi_{0} e^{\beta \varphi_{01}}}-V_{0}
$$




$$
V_{0}=\frac{m^{2}}{\beta^{2}}\left(e^{\beta\left(-2 \varphi_{01}+\varphi_{02}\right)}\left(1+\beta^{2} \chi_{0} \psi_{0} e^{\beta \varphi_{01}}\right)+e^{\beta\left(\varphi_{01}+\varphi_{0 n-1}\right)}+\sum_{k=2}^{n-1} e^{\beta\left(\varphi_{0 k-1}+\varphi_{0 k+1}-2 \varphi_{0 k}\right)}-n\right)
$$

where $\varphi_{0 n}=0$. The other Weyl group elements $w_{\alpha_{i}}$ and their combinations lead to transformations similar to (2.42), thus producing new families of classically equivalent integrable models $\mathcal{L}_{w_{\alpha_{i}}}$. Hence we can conclude that $P \Pi$-transformation given by eqns. (2.41) is the unique (affine) Weyl group transformation leaving (1.1) invariant. Contrary to the abelian affine Toda theories all the other Weyl transformations (different from $\Pi$ ) are not symmetries of (1.1), thus giving rise to new phenomena- Weyl families of IM's.

\section{Soliton Equations}

3.1 Vacua Backlund Transformations. Consider two arbitrary solutions $D_{1}$ and $D_{2}$ of eqns. (2.10). The corresponding Lax connections $\mathcal{A}_{\mu}^{W}(s)=\mathcal{A}_{\mu}^{W}\left(D_{s}\right), s=1,2$ are related by appropriate dressing (gauge) transformations $\Theta_{ \pm}$:

$$
\mathcal{A}_{\mu}^{W}(2)=\Theta_{ \pm} \mathcal{A}_{\mu}^{W}(1) \Theta_{ \pm}^{-1}+\left(\partial_{\mu} \Theta_{ \pm}\right) \Theta_{ \pm}^{-1}
$$

They leave invariant eqn. (2.2) together with the linear problem

$$
\left(\partial_{\mu}-\mathcal{A}_{\mu}^{W}\left(D_{s}\right)\right) T\left(D_{s}\right)=0 .
$$

The relation between the monodromy matrices $T_{s}=T\left(D_{s}\right)=P \exp \left(\oint \mathcal{A}_{\mu}^{W} d x^{\mu}\right)$ has the well known form 18

$$
T_{2}=\Theta_{ \pm} T_{1}, \quad \Theta_{+} T_{1}=\Theta_{-} T_{1} g_{0}^{(1)}
$$

where $g_{0}^{(1)}$ is a constant element of the corresponding affine group. The strategy in deriving the infinitesimal Backlund transformations $D_{1} \longleftrightarrow D_{2}$ consists in the following a) First solve eqn. (3.2) for $\Theta_{ \pm}\left(D_{s}\right)$ and $\mathbf{b}$ ) find first order differential equations for $D_{s}$ by substituting these $\Theta_{ \pm}\left(D_{s}\right)$ in (3.1). In the case of $A_{n}^{(1)}$ - Abelian affine Toda theories [25], and for all non-abelian Toda theories based on $A_{1}^{(1)}$, the realization of the above recipe is quite straightforward. We find that

$$
\Theta_{+}=X\left(1+\left(D_{1} X\right)^{-1} Y D_{2}\right)
$$

and the corresponding Backlund transformations take the following compact form

$$
\begin{aligned}
D_{1}^{-1} \partial D_{1} X-X D_{2}^{-1} \partial D_{2} & =\left[D_{1}^{-1} Y D_{2}, \epsilon_{-}\right] \\
\left(\bar{\partial} D_{1}\right) D_{1}^{-1} Y-Y\left(\bar{\partial} D_{2}\right) D_{2}^{-1} & =\left[D_{1} X D_{2}^{-1}, \epsilon_{+}\right]
\end{aligned}
$$

The constant elements $X\left(\lambda, a_{i}\right), Y\left(\lambda, b_{i}\right)$ of the universal enveloping algebra of $\mathcal{G}_{n}^{(a)}\left(A_{n}^{(1)}\right.$ or $A_{1}^{(1)}$ in our case), contain all the parameters $a_{i}, b_{i}$ of the Backlund transformation. They also have to satisfy the following conditions

$$
\left[X, \epsilon_{-}\right]=0, \quad\left[Y, \epsilon_{+}\right]=0
$$


It is not difficult to check that the second order equations $(2.10)$ are indeed the integrability conditions for the first order equations (3.5) if requirements (3.6) are fullfiled. The verification of the above statement does not depend on the explicit form of $\epsilon_{ \pm}$( and on the parametrization of $D_{s}$ ) and therefore is valid for our dyonic $A_{n}^{(1)}$ model (i.e. $\epsilon_{ \pm}$and $D_{s}$ given by eqns. (2.5) and (2.6)). It turns out however that the simple form (3.4) of $\Theta_{+}$takes place only for specific $g_{0}^{(1)}$ giving rise to 1 -soliton solutions [22], i.e. when $D_{1}=e^{i \beta_{0} \lambda_{1} \cdot H}=$ const . The derivation of the explicit form of the vacua Backlund transformation $\left(D_{1}=\right.$ const.) for our model (1.1) includes one more complication. Taking $X$ and $Y$ in the form

$$
\begin{aligned}
& X=X_{01} I+n X_{02} \lambda_{1} \cdot H+\sum_{k=1}^{n-1} a_{k}\left(\epsilon_{-}\right)^{k}, \\
& Y=Y_{01} I+n Y_{02} \lambda_{1} \cdot H+\sum_{k=1}^{n-1} b_{k}\left(\epsilon_{+}\right)^{k}
\end{aligned}
$$

and $D$ as in eqn. (2.6) we have to further impose on eqns. (3.5) the requirement of $\Pi$ symmetry (2.41) in order to get a complete system of equations. The result is

$$
\begin{aligned}
\partial \varphi_{k} & =\frac{m \gamma}{\beta}\left(e^{\beta\left(\varphi_{k-1}-\varphi_{k}+\frac{R}{n}\right)}-e^{\beta\left(\varphi_{n-1}+\frac{R}{n}\right)}+\frac{n-k}{n} \beta^{2} \psi \chi e^{-\beta \frac{R}{n}}\right) \\
\bar{\partial} \varphi_{k} & =\frac{m}{\gamma \beta}\left(e^{\beta\left(\varphi_{1}-\frac{R}{n}\right)}-e^{\beta\left(\varphi_{k+1}-\varphi_{k}-\frac{R}{n}\right)}+\frac{k}{n} \beta^{2} \psi \chi e^{-\beta \frac{R}{n}}\right)
\end{aligned}
$$

$k=1,2, \cdots, n-1, \varphi_{0}=\varphi_{n}=0$

$$
\begin{aligned}
\partial R & =-m \gamma \beta \psi \chi e^{-\beta \frac{R}{n}}, \quad \bar{\partial} R=\frac{m \beta}{\gamma} \psi \chi e^{-\beta \frac{R}{n}} \\
\partial \psi & =-m \gamma \psi e^{\beta\left(\varphi_{n-1}+\frac{R}{n}\right)}\left(1-\frac{n-1}{2 n} \beta^{2} \psi \chi e^{-\beta\left(\varphi_{n-1}+\frac{2 R}{n}\right)}\right) \\
\partial \chi & =-m \gamma \chi e^{\beta\left(\varphi_{1}-\frac{R}{n}\right)} \Delta, \quad \bar{\partial} \psi=\frac{m}{\gamma} \psi e^{\beta\left(\varphi_{1}-\frac{R}{n}\right)} \Delta \\
\bar{\partial} \chi & =\frac{m}{\gamma} \chi e^{\beta\left(\varphi_{n-1}+\frac{R}{n}\right)}\left(1-\frac{n-1}{2 n} \beta^{2} \psi \chi e^{-\beta\left(\varphi_{n-1}+\frac{2 R}{n}\right)}\right)
\end{aligned}
$$

where $\gamma=\frac{b_{2} \lambda}{a_{1}}=\frac{b_{1}}{X_{01}-X_{02}}=\frac{Y_{01}-Y_{02}}{a_{2}}=\cdots=e^{-b}$ is the Backlund transformation parameter and the following chain of algebraic relations should take place,

$$
\begin{aligned}
e^{-\beta \phi_{1}}-e^{\beta \phi_{2}} & =e^{-\beta \phi_{2}}-e^{\beta \phi_{3}}=\cdots=e^{-\beta \phi_{k}}-e^{\beta \phi_{k+1}} \\
=e^{-\beta \phi_{n-1}}-e^{\beta \phi_{n}} & =e^{-\beta \phi_{n}}-e^{\beta \phi_{1}}-\beta^{2} \tilde{\psi} \tilde{\chi}
\end{aligned}
$$

We have introduced new variables $\phi_{k}, \tilde{\psi}$ and $\tilde{\chi}$ defined by

$$
\begin{aligned}
\phi_{k} & =\varphi_{k}-\varphi_{k-1}-\frac{R}{n}, k=1, \cdots, n, \varphi_{0}=\varphi_{n}=0 \\
\tilde{\psi} & =e^{-\frac{\beta R}{2 n}} \psi, \quad \tilde{\chi}=e^{-\frac{\beta R}{2 n}} \chi, \quad \phi_{1}+\phi_{2}+\cdots \phi_{n}=-R
\end{aligned}
$$


in order to make evident the paralel with the abelian affine Toda case [25]. The algebraic eqns. (3.8) are crucial in the proof of the statement that the second order differential eqns. (2.1) are the integrability conditions for the first order system (3.7). In the case of the $A_{n-1}^{(1)}$ abelian affine Toda model $(\tilde{\psi}=\tilde{\chi}=0)$ the analog of eqns. (3.8) appears again as a result of the requirement of the abelian analog of the $\Pi$ - symmetry (2.41), i.e. $\left(\phi_{k}^{\prime}=-\phi_{n-k+1}\right)$ of the first order equations. They do not play however the same role as eqns. (3.8) in the non-abelian model (1.1), but are indeed essential in the derivation of 1-soliton solutions 8 25.

It is important to mention that, although our first order system (3.7) has rather complicated form ( including the non local field $R$ ) it can be obtained from the simple solitonic equations of the vector model (5.1) and (5.2) applying the integrated form of T-duality transformation (2.15). The same is indeed true for the corresponding 1-soliton solutions.

3.2 Soliton Spectrum. The main virtue of the vacua Backlund transformation $\left(D_{1}=\right.$ const. ) (3.5) is to provide an elementay proof [25] of the topological character of the soliton energy and momenta ( and also of the electric charge and the spins, in our case). The key point is that the derivation of the particle -like soliton spectrum $\left(M, Q_{e l}, Q_{\theta}, Q_{k}^{t o p}, s\right)$ does not require the explicit knowledge of the 1-soliton solutions. The conserved charges depend only on the asymptotics of the fields at $x \longrightarrow \pm \infty$ and the specific "solitonic conservation laws" "9 encoded in eqns. (3.7). In order to extend the arguments used in the abelian Toda model [25] to the $A_{n}^{(1)}$-dyonic model (1.1) it is convenient to rewrite eqns. (3.7) in terms of variables $\phi_{p}, \tilde{\psi}$ and $\tilde{\chi}$ defined in $(3.9)$

$$
\begin{aligned}
\partial \phi_{p} & =\gamma \frac{m}{\beta}\left(e^{-\beta \phi_{p}}-e^{-\beta \phi_{p-1}}+\beta^{2} \tilde{\psi} \tilde{\chi} \delta_{p, 1}\right) \\
\bar{\partial} \phi_{p} & =\frac{m}{\gamma \beta}\left(e^{\beta \phi_{p}}-e^{\beta \phi_{p+1}}-\beta^{2} \tilde{\psi} \tilde{\chi} \delta_{p, n}\right), p=1,2, \cdots, n \\
\partial \tilde{\psi} & =-\gamma m \tilde{\psi} e^{-\beta \phi_{n}} \Delta_{n}, \quad \partial \tilde{\chi}=-\gamma m \tilde{\chi} e^{\beta \phi_{1}} \Delta_{1} \\
\bar{\partial} \tilde{\psi} & =\frac{m}{\gamma} \tilde{\psi} e^{\beta \phi_{1}} \Delta_{1}, \quad \bar{\partial} \tilde{\chi}=\frac{m}{\gamma} \tilde{\chi} e^{-\beta \phi_{n}} \Delta_{n}
\end{aligned}
$$

where $\Delta_{1}=1+\frac{1}{2} \beta^{2} \tilde{\psi} \tilde{\chi} e^{-\beta \phi_{1}}, \quad \Delta_{n}=1-\frac{1}{2} \beta^{2} \tilde{\psi} \tilde{\chi} e^{\beta \phi_{n}}$. We next observe that the first order system (3.10) (and (3.7) as well) admits the following "solitonic" conservation laws :

- non chiral

$$
\begin{aligned}
& \bar{\partial}\left(\gamma e^{-\beta \phi_{k}}\right)+\partial\left(\frac{1}{\gamma} e^{\beta \phi_{k+1}}\right)=0, k=1, \cdots, n-1 \\
& \bar{\partial}\left(\gamma e^{-\beta \phi_{n}} \Delta_{n}\right)+\partial\left(\frac{1}{\gamma} e^{\beta \phi_{1}} \Delta_{1}\right)=0
\end{aligned}
$$

- chiral

$$
\left(\gamma \bar{\partial}+\frac{1}{\gamma} \partial\right) e^{ \pm \beta \phi_{p}}=0, \quad p=1,2, \cdots, n, \quad\left(\gamma \bar{\partial}+\frac{1}{\gamma} \partial\right)(\tilde{\psi} \tilde{\chi})=0,
$$

\footnotetext{
8 in ref. 25] they have been used in the form of first integrals $e^{-\beta \phi_{k}}-e^{\beta \phi_{k+1}}=$ const.

${ }^{9}$ The precise statement is that the soliton spectrum is determined by the boundary conditions (b.c. ) and by the algebra of symmetries of the first order (BPS-like) equations (3.7)
} 


$$
\left(\gamma \bar{\partial}-\frac{1}{\gamma} \partial\right) \ln \left(\frac{\tilde{\psi}}{\tilde{\chi}}\right)=0
$$

Note that the algebraic relations (3.8) have been used in the derivation of the chiral conservation currents $(3.12)$ and $(3.13)$. The conclusion is that the complete algebra of symmetries of the 1-soliton solutions of the axial model (1.1) is generated by the Poincaré currents $T_{\mu \nu}$ (with "charge" the 2-momenta $P_{\mu}$ ) and $M^{\rho, \mu \nu}=x^{\mu} T^{\rho \nu}-x^{\nu} T^{\rho \mu}+$ spin matrix ( the 2-d spin-orbital momenta is $i \int_{-\infty}^{\infty} d x M^{0,01}=s$ ), the electric and magnetic currents $J_{e l}^{\mu, a x}, J_{\theta}^{\mu, a x}, J_{k}^{\mu}$ given by eqns.(2.24),(2.25) and (2.26) and by the "internal" currents (3.11), (3.12) and (3.13). Leaving aside an interesting problem of deriving the explicit form of this algebra, we will restrict ourselves to consider few simple consequences of eqn. (3.11) and (3.12), that allows us to find the mass spectrum of the $U(1)$ charged 1 -solitons.

Taking into account eqns. (3.10) and (3.11) we realize that the potential (2.18)

$$
V=\frac{m^{2}}{\beta^{2}}\left(\sum_{k=1}^{n} e^{\beta\left(\phi_{k+1}-\phi_{k}\right)}+\beta^{2} \tilde{\psi} \tilde{\chi} e^{-\beta \phi_{n}}-n\right)
$$

and the stress-tensor $T_{\mu \nu}$ :

$$
\begin{aligned}
& T_{00}=\frac{1}{4} \sum_{k=1}^{n}\left(\left(\partial \phi_{k}\right)^{2}+\left(\bar{\partial} \phi_{k}\right)^{2}\right)+\frac{e^{-\beta \phi_{1}}}{2 \Delta_{1}}(\bar{\partial} \tilde{\psi} \bar{\partial} \tilde{\chi}+\partial \tilde{\psi} \partial \tilde{\chi})+V \\
& T_{01}=\frac{1}{4} \sum_{k=1}^{n}\left(\left(\partial \phi_{k}\right)^{2}-\left(\bar{\partial} \phi_{k}\right)^{2}\right)+\frac{e^{-\beta \phi_{1}}}{2 \Delta_{1}}(\partial \tilde{\psi} \partial \tilde{\chi}-\bar{\partial} \tilde{\psi} \bar{\partial} \tilde{\chi})
\end{aligned}
$$

are total derivatives:

$$
V=-\frac{1}{2}\left(\bar{\partial} F^{-}+\partial F^{+}\right), \quad T_{00}=\partial_{x}\left(F^{-}-F^{+}\right), \quad T_{01}=\partial_{x}\left(F^{-}+F^{+}\right)
$$

The $F^{ \pm}$we have introduced in eqn. (3.15) turn out to be the light cone components $F_{\mu}=$ $\left(F^{-}, F^{+}\right)$of certain linear combination of the conserved currents (3.11):

$$
F^{-}=-\frac{m \gamma}{\beta^{2}} \sum_{k=1}^{n} e^{-\beta \phi_{k}}, \quad F^{+}=\frac{m}{\gamma \beta^{2}}\left(\sum_{k=1}^{n} e^{\beta \phi_{k}}+\beta^{2} \tilde{\psi} \tilde{\chi}\right)
$$

Hence the energy and the momentum of the 1-soliton decribed by eqns. (3.10) receive contributions from the boundary terms only:

$$
E=\int_{-\infty}^{\infty} T_{00} d x=\left.\left(F^{-}-F^{+}\right)\right|_{-\infty} ^{\infty}, \quad P=\int_{-\infty}^{\infty} T_{01} d x=\left.\left(F^{-}+F^{+}\right)\right|_{-\infty} ^{\infty}
$$

The same is true for the electric charge (2.24), (2.30)

$$
\begin{gathered}
Q_{e l}^{a x}=\int_{-\infty}^{\infty} J_{e l}^{0, a x} d x=2 \beta_{0}(R(\infty)-R(-\infty)) \\
\int_{-\infty}^{\infty} J_{e l, i m p r}^{0, a x} d x=Q_{e l}^{a x}-\frac{\beta_{0}^{3}}{4 \pi^{2}} \sum_{k=1}^{n-1} \nu_{k}\left(\varphi_{k}(\infty)-\varphi_{k}(-\infty)\right)
\end{gathered}
$$


as well as for the topological currents $J_{\theta}^{\mu, a x}$ and $J_{k}^{\mu}$ by construction. We fix the asymptotics of the fields $\phi_{p}, \tilde{\chi}, \tilde{\psi}$ ( and $R$ ) at $x \longrightarrow \pm \infty$ by requiring $\left.V\right|_{x \rightarrow \pm \infty}=0$, i.e. the solutions of (3.10) we seek for to interpolate between two nontrivial vacua (2.23). More precisely, we choose,

$$
\varphi_{l}( \pm \infty)=\frac{2 \pi}{\beta_{0}} \frac{l}{n} N_{ \pm}, \quad l=1,2, \cdots, n-1, \quad \tilde{\chi} \tilde{\psi}( \pm \infty)=0, \quad R( \pm \infty)=\frac{2 \pi}{\beta_{0}} f_{ \pm}
$$

and 10

$$
\theta( \pm \infty)=-\left.\frac{i}{2 \beta_{0}} \ln \left(\frac{\tilde{\chi}}{\tilde{\psi}}\right)\right|_{ \pm \infty}=\frac{\pi}{2 \beta_{0}} L_{ \pm}
$$

where $N_{ \pm}$and $L_{ \pm}$are arbitrary integers and $f_{ \pm}$are real numbers. According to eqn. (3.9) we also get

$$
\phi_{p}( \pm \infty)=\frac{2 \pi}{n \beta_{0}}\left(N_{ \pm}-f_{ \pm}\right)
$$

Substituting (3.20) into (3.17) for $\beta=i \beta_{0}$ we derive the 1 -soliton energy momentum spectrum ए11:

$$
\begin{gathered}
E=\frac{4 m}{\beta_{0}^{2}} n \sin \frac{1}{4 n}\left(Q_{e l}^{a x}-\beta_{0}^{2} Q_{m a g}\right) \sin a \\
P=\frac{4 m i}{\beta_{0}^{2}} n \sin \frac{1}{4 n}\left(Q_{e l}^{a x}-\beta_{0}^{2} Q_{m a g}\right) \cos a
\end{gathered}
$$

where $a=\frac{\pi}{n}\left(N_{+}+N_{-}-f_{+}-f_{-}\right)-i b, \gamma=e^{-b}$. The soliton charges are given by:

$$
Q_{e l}^{a x}=4 \pi\left(f_{+}-f_{-}\right), \quad j_{\varphi}=N_{+}-N_{-}=0, \pm 1, \pm 2, \cdots, \pm(n-1), \quad Q_{m a g}=\frac{4 \pi}{\beta_{0}^{2}} j_{\varphi}
$$

It turns out that the masses of the charged 1-solitons of the axial model (1.1):

$$
M^{a x}=\sqrt{E^{2}-P^{2}}=\frac{4 m}{\beta_{0}^{2}} n\left|\sin \frac{1}{4 n}\left(Q_{e l}^{a x}-\beta_{0}^{2} Q_{m a g}\right)\right|
$$

does not depend on the topological charge $Q_{\theta}^{a x}$. As we shall show in Sect.5 the same formula (3.22) takes place for the 1-solitons of the vector model (1.3) but with $Q_{e l}^{a x}$ replaced by its dual $Q_{\tilde{\theta}}^{v e c}=-i \int_{-\infty}^{\infty} \partial_{x} \ln \left(A^{2}\right)$ and $Q_{e l}^{v e c}=-i \int_{-\infty}^{\infty} \partial_{x} \ln \left(\tilde{u}^{2}\right)=0$. Note that the 1 -soliton spectrum of the dyonic model (1.1) (i.e., $\left.M, Q_{e l}, Q_{\theta}, Q_{k}, s, E\right)$ depends upon arbitrary parameters $f_{ \pm}$ (i.e. on the b.c. of the nonlocal field $R$ ). They are not related to the b.c. of the physical fields $\varphi_{l}, \psi, \chi$ and it does not seems to be one of the new internal conserved charges. We

${ }^{10}$ The precise definition [22] of the topological charge $Q_{\theta}$ for arbitrary complex $\psi$ and $\chi$ is $Q_{\theta}=$ $\operatorname{Re}(\theta(\infty)-\theta(-\infty)), \quad \operatorname{Re} \theta( \pm \infty)=\left.\frac{1}{2 \beta_{0}}(\operatorname{Arg} \tilde{\chi}-\operatorname{Arg} \tilde{\psi})\right|_{ \pm \infty}$.For the 1-soliton solutions it coincides with (3.19)

11 The complex form of $E$ and $P$ is misleading. It will be shown below that the chain relations (3.8) together with the conservation laws (3.11), 3.12 and (3.13) impose conditions on $N_{ \pm}$and $f_{ \pm}$that ensures the reality of $E$ and $P,(E \geq 0$, as well $)$ 
are going to show now that $f_{ \pm}$represent the value of the following chain of first integrals of eqn. (3.10)

$$
y=e^{-i \beta_{0} \phi_{p}}-e^{i \beta_{0} \phi_{p+1}}=e^{-i \beta_{0} \phi_{n}}-e^{i \beta_{0} \phi_{1}}+\beta_{0}^{2} \tilde{\psi} \tilde{\chi}, \quad p=1, \cdots, n-1
$$

It is easy to check that as a consequence of eqns. (3.8), (3.11) and (3.12) we have

$$
\bar{\partial}\left(e^{-i \beta_{0} \phi_{1}}-e^{i \beta_{0} \phi_{2}}\right)=0, \quad \bar{\partial}\left(e^{-i \beta_{0} \phi_{n}}-e^{i \beta_{0} \phi_{1}}+\beta_{0}^{2} \tilde{\psi} \tilde{\chi}\right)=0, \quad \text { etc }
$$

Combining them with the chiral conservation laws (3.11) and (3.12) we realize that all the members of the chain relations (3.8) represent first integrals of the system (3.23). Thus, the only ("zero") mode of the double chiral (i.e., $\partial y=\bar{\partial} y=0$ ) conserved currents $y=2 i \sin \alpha$ describes an internal conserved charge. Since (3.23) is valid for all $x$ and $t$ applying them to the boundary case ( $t$ fixed and $x \rightarrow \pm \infty$ ) we find the following relations between $f_{ \pm}, N_{ \pm}$ and $\alpha$ :

$$
\sin \frac{2 \pi}{n}\left(f_{ \pm}-N_{ \pm}\right)=\sin \alpha
$$

The general solution of (3.25) is given by

$$
\frac{2 \pi}{n}\left(f_{ \pm}-N_{ \pm}\right)=\alpha+2 \pi S_{ \pm}^{(a)}
$$

or

$$
\frac{2 \pi}{n}\left(f_{ \pm}-N_{ \pm}\right)=\operatorname{Sign}(\alpha) \pi-\alpha+2 \pi S_{ \pm}^{(b)}
$$

$S_{ \pm}^{(a, b)}=0, \pm 1, \pm 2, \cdots$. We chose $f_{ \pm}$(for $\cos \alpha>0$ ), such that they provide a nontrivial $\alpha$-dependence of $Q_{e l}$ :

$$
\begin{aligned}
& f_{+}=\frac{n}{2 \pi}\left(\alpha+2 \pi S_{+}^{(a)}\right)+N_{+} \\
& f_{-}=-\frac{n}{2 \pi}\left(\alpha-\pi \operatorname{Sign}(\alpha)+2 \pi S_{-}^{(b)}\right)+N_{-}
\end{aligned}
$$

(and $f_{+} \leftrightarrow f_{-}, \quad N_{+} \leftrightarrow N_{-}$for $\left.\cos \alpha<0\right)$ and therefore

$$
\begin{aligned}
& Q_{e l}^{a x}=4 \pi\left(f_{+}-f_{-}\right)=4 n\left(\alpha-\frac{\pi}{2} \operatorname{Sign}(\alpha)\right)+4 \pi j_{\varphi}, \\
& \int_{\infty}^{\infty} J_{e l, i m p r}^{0, a x} d x=Q_{e l}^{a x}-\frac{\nu \beta_{0}^{2}}{2 \pi} j_{\varphi}
\end{aligned}
$$

neglecting the term $n\left(S_{+}^{(a)}+S_{-}^{(b)}\right)$ in $Q_{e l}^{a x}$ since $j_{\varphi}$ is defined as integer $\bmod n$.

Taking into account $(3.28)$ we realize that

$$
\sin a=-\operatorname{Sign}(\alpha) \cosh b, \quad \cos a=-i \operatorname{Sign}(\alpha) \sinh b
$$

and therefore

$$
E=M^{a x} \cosh b, \quad P=-M^{a x} \sinh b, \quad M^{a x}=\frac{4 m n}{\beta_{0}^{2}}|\cos \alpha|
$$


(i.e., $E \geq 0$ as it should be ). To make the discussion of the spectrum of the $U(1)$-charged topological soliton complete we antecipate the semiclassical quantization of $Q_{e l}$ (see Sect. 7.1 below):

$$
Q_{e l}^{a x}=\beta_{0}^{2}\left(j_{e l}+\frac{\nu}{2 \pi} j_{\varphi}\right), \quad j_{e l}=0, \pm 1, \cdots
$$

This form of $Q_{e l}^{a x}$ confirms the arguments presented in Sect. 2 that the electric charge of the solitons (and breathers as well [22]) of the axionic model (1.1) gets contributions from the magnetic charge $Q_{m a g}=\frac{4 \pi}{\beta_{0}^{2}} j_{\varphi}$. It is important to note that the 1 -soliton charges $\left(Q_{e l}^{a x}, Q_{m a g}\right)$ (3.30) coincide with the electric and magnetic charges of the dyonic solutions of 4-D $S U(n+1) \mathrm{YMH}$ model with CP -breaking $\theta$-term [29]. The 2-D soliton mass spectrum (1.5) however is different from the semiclassical masses $M_{d y o n}^{2}=<\phi>\left(Q_{e l}^{2}+Q_{m a g}^{2}\right)$ of 4 -D YMH dyons. It is worthwhile to mention that the $n \rightarrow \infty$ limit of our mass formula (3.22) coincides with the BPS bounds for the masses of particular dyons $\left(Q_{e l}^{(2)}=0=Q_{m a g}^{(1)}\right)$ of four dimensional $N=2$ Supersymmetric YM theory [21].

\section{Electrically Charged Topological Solitons}

4.1 Soliton Solutions. In our derivation of the dyonic properties (1.5) of the 1-solitons of (1.1) we left unanswered the important question: whether eqs. (2.1) and (3.10) possess soliton solutions with both charges $Q_{e l}$ and $Q_{m}$ different from zero, i.e. $N_{+} \neq N_{-}$and $f_{+} \neq f_{-}$. It is indeed the case as we will show by explicit construction of solutions of eqns. (3.10) and (3.8). We first consider the $\phi_{p}, p=1, \cdots, n$ equations. Taking into account the algebraic relations $(3.8)$ and $(3.23)$ we realize that they can be rewritten in the following compact form:

$$
\begin{aligned}
& \partial\left(e^{i \beta_{0} \phi_{p}}\right)=m e^{-b}\left(1-e^{2 i \beta_{0} \phi_{p}}-2 i \sin \alpha e^{i \beta_{0} \phi_{p}}\right) \\
& \bar{\partial}\left(e^{i \beta_{0} \phi_{p}}\right)=-m e^{b}\left(1-e^{2 i \beta_{0} \phi_{p}}-2 i \sin \alpha e^{i \beta_{0} \phi_{p}}\right)
\end{aligned}
$$

We next introduce the "solitonic" variables

$$
\begin{array}{ll}
\rho_{+}=\cosh (b) x-\sinh (b) t, & \partial_{\rho_{+}}=\sinh (b) \partial_{t}+\cosh (b) \partial_{x} \\
\rho_{-}=\cosh (b) t-\sinh (b) x, & \partial_{\rho_{-}}=\cosh (b) \partial_{t}+\sinh (b) \partial_{x}
\end{array}
$$

and taking into account the chiral conservation laws (3.12)-(3.13) we get

$$
\partial_{\rho_{+}}\left(e^{i \beta_{0} \phi_{p}}\right)=m\left(1-e^{2 i \beta_{0} \phi_{p}}-2 i \sin (\alpha) e^{i \beta_{0} \phi_{p}}\right), \quad \partial_{\rho_{-}}\left(e^{i \beta_{0} \phi_{p}}\right)=0
$$

The general solution of eqns. (4.1) is given by

$$
e^{i \beta_{0} \phi_{p}}=e^{i \alpha} \frac{S_{p} e^{-2 i \alpha+2 m \cos \alpha \rho_{+}}-1}{S_{p} e^{2 m \cos \alpha \rho_{+}}+1}
$$

where $S_{p}$ are certain integration constants satisfying the recursive relations

$$
S_{p+1}=e^{-2 i \alpha \pm i \pi} S_{p}
$$


as a consequence of eqns. (3.23) and (4.2). Therefore

$$
S_{p}=(-1)^{p-1} e^{-2 i \alpha(p-1)} S_{1} \equiv(-1)^{p-n} e^{-2 i \alpha(p-n)} \delta
$$

and eqn. (4.2) acquires the final form

$$
e^{i \beta_{0} \phi_{p}}=e^{i \alpha} \frac{(-1)^{p-1} S_{1} e^{-2 i \alpha p} e^{2 m \cos \alpha \rho_{+}}-1}{(-1)^{p-1} S_{1} e^{-2 i \alpha(p-1)} e^{2 m \cos \alpha \rho_{+}}+1}
$$

With eqn. (4.3) at hand we can write the 1-solitons in the original variables $\varphi_{l}$ and $R$,

$$
R=-\sum_{p=1}^{n} \phi_{p}, \quad \varphi_{l}=\frac{l}{n} R+\sum_{p=1}^{l} \phi_{p}
$$

as follows

$$
\begin{gathered}
e^{i \beta_{0} R}=e^{-i n(\alpha-\pi \operatorname{Sign}(\alpha))} \frac{e^{-f}+(-1)^{n} e^{2 i n \alpha} e^{f}}{e^{-f}+e^{f}} \\
e^{i \beta_{0} \varphi_{l}}=\frac{e^{-f}+(-1)^{l-n} e^{2 i \alpha(n-l)} e^{f}}{\left(e^{f}+e^{-f}\right)^{\frac{l}{n}}\left(e^{-f}+(-1)^{n} e^{2 i \alpha n} e^{f}\right)^{\frac{n-l}{n}}}, \quad l=1, \cdots n-1
\end{gathered}
$$

where $\delta$ is a complex constant, $f=m \rho_{+} \cos \alpha+\frac{1}{2} \ln (\delta)$. The next step is to derive solutions for $\psi$ and $\chi$. Eqns. (4.5) and (4.6) together with the algebraic relation (3.23) allows us to determine the product $\psi \chi$ :

$$
\begin{aligned}
\beta^{2} \psi \chi & =N^{2}\left(e^{f}+e^{-f}\right)^{-\frac{n+1}{n}}\left(e^{-f}+(-1)^{n} e^{2 i \alpha n} e^{f}\right)^{-\frac{n-1}{n}} \\
N^{2} & =\left(1+e^{-2 i \alpha}\right)\left((-1)^{n} e^{2 i \alpha n}-1\right)
\end{aligned}
$$

It turns out that the ratio $\frac{\tilde{\psi}}{\tilde{\chi}}=\frac{\psi}{\chi}$ satisfies the following simple equations

$$
\partial \ln \left(\frac{\psi}{\chi}\right)=m e^{-b}\left(e^{i \beta_{0} \phi_{2}}-e^{-i \beta_{0} \phi_{1}}\right), \quad \bar{\partial} \ln \left(\frac{\psi}{\chi}\right)=m e^{b}\left(e^{i \beta_{0} \phi_{2}}-e^{-i \beta_{0} \phi_{1}}\right)
$$

Applying once more eqn. (3.23) we find that $\ln \left(\frac{\psi}{\chi}\right)$ is independent of $\rho_{+}$while the $\rho_{-}$dependence is linear

$$
\partial_{\rho_{-}} \ln \left(\frac{\psi}{\chi}\right)=-2 i m \sin \alpha
$$

Therefore the ratio $\frac{\psi}{\chi}$ is given by

$$
\frac{\psi}{\chi}=e^{-2 i\left(m \sin \alpha \rho_{-}+q\right)}
$$

where $q$ is an arbitrary complex constant. Thus, eqns. (4.7) and (4.9) completely determine the solution of the first order equations (3.7)

$$
\begin{aligned}
& \psi= \pm \frac{N}{\beta} e^{-i\left(m \sin \alpha \rho_{-}+q\right)}\left(e^{f}+e^{-f}\right)^{-\frac{n+1}{2 n}}\left(e^{-f}+(-1)^{n} e^{2 i \alpha n} e^{f}\right)^{-\frac{n-1}{2 n}} \\
& \chi= \pm \frac{N}{\beta} e^{i\left(m \sin \alpha \rho_{-}+q\right)}\left(e^{f}+e^{-f}\right)^{-\frac{n+1}{2 n}}\left(e^{-f}+(-1)^{n} e^{2 i \alpha n} e^{f}\right)^{-\frac{n-1}{2 n}}
\end{aligned}
$$


The 1-soliton solution of the dyonic model (1.1) is presented by the set of functions (of $\rho_{+}$ and $\left.\rho_{-}\right)$(4.6) and (4.10).

4.2 Soliton Charges. It remains to be shown that these solitons carry non trivial electric and magnetic charges. The simplest way to do this consists in calculating the asymptotics of the fields $R, \varphi_{l}, \psi, \chi$ from eqns. (4.5), (4.6) and (4.10) and further comparing with the values (3.19) and 3.28) proposed in Sect. 3. We have to distinguish three cases [7: i) $\cos \alpha>0$; ii) $\cos \alpha<0$; iii) $\sin \alpha=0, n$ odd. Taking the limit $x \longrightarrow \pm \infty$ in eqns. (4.5), (4.6) and (4.10) we obtain for $\cos \alpha>0$ :

$$
\begin{aligned}
\varphi_{l}( \pm \infty) & =\frac{2 \pi}{\beta_{0}}\left(\frac{l}{n} N_{ \pm}^{l}+K_{ \pm}^{l}\right), \theta(-\infty)=\theta(\infty), \quad j_{\theta}=0 \\
R(\infty) & =\frac{2 \pi}{\beta_{0}} f_{+}=\frac{2 \pi}{\beta_{0}}\left(\frac{n \alpha}{2 \pi}+S_{+}\right), \\
R(-\infty) & =\frac{2 \pi}{\beta_{0}} f_{-}=\frac{2 \pi}{\beta_{0}}\left(-\frac{n(\alpha-\pi \operatorname{Sign}(\alpha))}{2 \pi}+S_{-}\right),
\end{aligned}
$$

The arbitrary integers $N_{ \pm}^{l}, K_{ \pm}^{l}$ and $S_{ \pm}$are further restricted by the conditions

1. to provide nontrivial zeros of the potential (2.18)

2. chain relations (3.8) and (3.23)

The simplest solution satisfying 1) and 2) that leads to nontrivial dyonic spectra (3.29) (with $\left.j_{\theta}=0\right)$ is given by

$$
N_{ \pm}^{l}=N_{ \pm}(\bmod n), \quad S_{ \pm}=N_{ \pm}(\bmod n), \quad K_{ \pm}^{l}=0
$$

for all $l=1,2, \cdots, n-1$ ( $N_{ \pm}$being new arbitrary integers). The important fact is that the 1-soliton solution given by (4.5), (4.6) and (4.10) (for $\cos \alpha>0$ ) are topological and electrically charged, i.e., with both charges $Q_{e l}^{a x}$ and $Q_{m}$ different from zero. Such dyonic type soliton combines the properties of the Lund-Regge (complex SG) solitons [11] with the $A_{n}$-abelian affine Toda solitons [17, [25]. The case $\cos \alpha<0$ leads again to (4.11) but with $N_{+}^{l} \leftrightarrow N_{-}^{l}$ and $f_{+} \leftrightarrow f_{-}$, i.e. $Q_{e l} \rightarrow-Q_{e l}$ and $Q_{m a g} \rightarrow-Q_{m a g}$. Therefore the corresponding "particles " can be interpreted as antisolitons.

4.3 Periodic lumps. The main feature of the 1 -solitons with $\sin \alpha \neq 0($ and $\cos \alpha \neq 0)$ is that in the rest frame $v=\tanh b=0$, they represent periodic in time $t,\left(\tau=\frac{2 \pi}{\omega}, \omega=m \sin \alpha\right)$ bounded solutions. Such "periodic lumps" behaviour is familiar from the CSG solitons [11] and the breathers ( doublet solutions ) of the SG theory [3]]. It reflects the angular property of the phase $2 i \beta_{0} \theta=\ln \left(\frac{\tilde{\chi}}{\psi}\right)$ of the fields $\psi, \chi$, (see eqn. 4.9$)$ ):

$$
\theta(t+\tau, x)=\theta(t, x)-\frac{2 \pi}{\beta_{0}}
$$

and therefore $t \in S_{1}$ and $\theta \in S_{1}$. The particle interpretation of the 1-soliton data: center of mass $X=R e \frac{1}{2} \ln (\delta), U(1)$ - moduli $q$, its velocity $v=\tanh (b)$ and the angular velocity

\footnotetext{
${ }^{12}$ We are not considering here two limiting cases: a) $\cos \alpha=0$ since there are no true 1 -soliton and b) $\sin \alpha=0, n$ even which leads to $N^{2}=0$ and $\psi=\chi=0$ (i.e. to the $A_{n}^{(1)}$ abelian Toda model)
} 
$\omega=m \sin \alpha$ can be borrowed from the CSG model [11] as particle with internal coordinate $q$, rotating in the $q$ space with constant angular velocity $\omega$ at the rest frame $v=0$. Another interpretation comes from the SG breather, as bounded motion of charged particles [31]. The singular case $\sin \alpha=0$, i.e. $\alpha=s \pi, \quad s=0, \pm 1, \pm 2, \cdots$ and $n$-odd (i.e. $N^{2} \neq 0$ ) is an example of static solitons $(v=0)$ with charges

$$
Q_{m a g}=\frac{4 \pi}{\beta_{0}^{2}} j_{\varphi}, \quad Q_{e l}^{a x}=4 n \pi\left(\left(s-\frac{1}{2}\right) \operatorname{sign}(\cos \alpha)+\frac{j_{\varphi}}{n}\right)
$$

and degenerate (independent of charges) mass $M=\frac{4 m}{\beta_{0}^{2}} n$.

4.4 Breathers. It is important to note that the electrically charged topological 1-soliton solution of our model do not exhaust all the particle-like solutions of (1.1). The complete list of (topologically ) stable 1-solitons, breathers and breathing ( or "excited solitons") includes together with the above constructed $U(1)$-charged topological 1-soliton (2-D dyon) also the neutral 1-soliton of specie $d, d=1, \cdots, n-1\left(M_{d}, Q_{m a g}=\frac{4 \pi}{\beta_{0}^{2}} d, Q_{e l}^{a x}=0, Q_{\theta}^{a x}=0\right.$, i.e. monopole ) and their bound states:

- The $A_{n-1}^{(1)}$ abelian affine Toda breather (two neutral vertices [17]) and the "excited solitons".

- The (NA Toda ) three vertex breathing [22] describing the one charged 1-soliton and one neutral $(d)$ 1-soliton bound state.

- The four vertex charged breathers [22] describing the bound state of two charged topological 1-solitons.

The explicit construction of all these solutions for the axial model (1.1) as well as their spectrum are presented in our forthcoming publication [22]. The method employed in [22] is a slight modification of the standard (abelian Toda) "vertex operators " 18] (or soliton specialization [17] or Hirota $\tau$ function[26]) method adapted to the case of the singular NonAbelian affine Toda models (1.1) and (1.3). The 3-vertex breather represent an interesting example of $U(1)$ charged particle-like solution carrying both topological charges $j_{\varphi}$ and $j_{\theta}= \pm 1$ (remember that $j_{\theta}=0$ for our charged solitons (4.5), (4.6), (4.10) ) [22]:

$$
\begin{aligned}
Q_{e l}^{a x} & =\beta_{0}^{2}\left(j_{e l}+\frac{\nu}{2 \pi}\left(j_{\varphi}+d\right)\right), \quad Q_{\text {mag }}=\frac{4 \pi}{\beta_{0}^{2}}\left(j_{\varphi}+d\right) \\
Q_{\theta} & =\frac{\pi}{2}\left(j_{\theta}+4 \tilde{\gamma} j_{\varphi}\right), \quad j_{\theta}= \pm 1 \quad ; j_{\varphi}, d= \pm 1, \cdots, \pm(n-1) .
\end{aligned}
$$

Due to the fact that $Q_{\theta}^{a x} \neq 0$ is the T-dual of the vector model electric charge $Q_{e l}^{v e c} \neq 0\left(Q_{e l}^{a x}\right.$ is the dual of the vector model topological charge $\left.Q_{\tilde{\theta}}^{v e c}=-2 i \int d x \partial_{x} \ln (A)\right)$, this solution plays an important role in the understanding of the T-duality relations between the discrete spectra of the axial and vecor IM's. 


\section{$5 \quad$ Vector Model Solitons}

5.1 First order Equations. Taking $D_{1}=D_{v}, D_{2}=e^{\beta \lambda_{1} \cdot H}$ and $X=X_{01} I+n X_{02} \lambda_{1} \cdot H+$ $\sum_{k=1}^{n-1} a_{k}\left(\epsilon_{-}\right)^{k}, \quad Y=Y_{01} I+n Y_{02} \lambda_{1} \cdot H+\sum_{k=1}^{n-1} b_{k}\left(\epsilon_{+}\right)^{k}$ in eqn. (3.5) we derive the following incomplete system of first order equations

$$
\begin{aligned}
\partial B & =m \gamma(1-A B) c_{1} c_{2} \cdots c_{n-1}, \quad \bar{\partial} A=-\frac{m}{\gamma} \frac{(1-A B)}{c_{1} c_{2} \cdots c_{n-1}} \\
\partial \ln \left(c_{1}\right) & =m \gamma\left(\frac{1}{c_{1}}-A c_{1} c_{2} \cdots c_{n-1}\right), \quad \bar{\partial} \ln \left(c_{1}\right)=\frac{m}{\gamma}\left(c_{1}-c_{2}\right) \\
\partial \ln \left(c_{k}\right) & =m \gamma\left(\frac{1}{c_{k}}-\frac{1}{c_{k-1}}\right), \quad \bar{\partial} \ln \left(c_{k}\right)=\frac{m}{\gamma}\left(c_{k}-c_{k+1}\right) \\
\partial \ln \left(c_{n-1}\right) & =m \gamma\left(\frac{1}{c_{n-1}}-\frac{1}{c_{n-2}}\right) \quad \bar{\partial} \ln \left(c_{n-1}\right)=\frac{m}{\gamma}\left(c_{n-1}-\frac{B}{c_{1} c_{2} \cdots c_{n-2}}\right)
\end{aligned}
$$

$k=2, \cdots, n-2$, where $\gamma=\frac{b_{2} \lambda}{a_{1}}=\frac{b_{1}}{\left(X_{01}-X_{02}\right)}=\cdots=e^{-b}$ is an arbitrary parameter related to the soliton velocity $v$. We next impose the condition of invariance of (5.1) under the discrete symmetries (2.39). It requires that the equations

$$
\bar{\partial} B=-\frac{m}{\gamma}(1-A B) c_{1} c_{2} \cdots c_{n-1}, \quad \partial A=m \gamma \frac{(1-A B)}{c_{1} \cdots c_{n-1}}
$$

and the following chain of algebraic relations

$$
A c_{1} c_{2} \cdots c_{n-1}-c_{1}=\frac{1}{c_{1}}-c_{2}=\cdots=\frac{1}{c_{n-2}}-c_{n-1}=\frac{1}{c_{n-1}}-\frac{B}{c_{1} c_{2} \cdots c_{n-1}}
$$

to be satisfied. An easy check confirms that each set of $A, B, c_{i}$ that solves (5.1) and (5.2) and

$$
\frac{B}{c_{1} c_{2} \cdots c_{n-1}}+A c_{1} c_{2} \cdots c_{n-1}=\frac{1}{c_{n-1}}+c_{1}
$$

satisfies the second order equations (2.9) as well. The remaining algebraic relations ensure the compatibility of (5.4) and (5.1), (5.2), i.e. they can be obtained by differentiating (5.4) and then simplifying with help of (5.1) and (5.2).

5.2 Solitons. An important property of the first order system (5.1) and (5.2) is the existence of specific "solitonic" conservation laws:

- nonchiral

$$
\begin{aligned}
\bar{\partial}\left(\gamma A c_{1} c_{2} \cdots c_{n-1}\right)+\partial\left(\frac{1}{\gamma} c_{1}\right) & =0, \quad \bar{\partial}\left(\frac{\gamma}{c_{n-1}}\right)+\partial\left(\frac{1}{\gamma} \frac{B}{c_{1} c_{2} \cdots c_{n-1}}\right)=0 \\
\bar{\partial}\left(\gamma c_{k}^{-1}\right)+\partial\left(\frac{1}{\gamma} c_{k+1}\right) & =0, \quad k=1,2, \cdots, n-1
\end{aligned}
$$


- chiral

$$
\begin{aligned}
& \left(\gamma \bar{\partial}+\frac{1}{\gamma} \partial\right) d=0, \quad d=A, B, c_{k} \\
& \left(\gamma \bar{\partial}-\frac{1}{\gamma} \partial\right) \tilde{u}=0, \quad \tilde{u}=\frac{u\left(c_{1} c_{2} \cdots c_{n-1}\right)^{\frac{1}{2}}}{(A B-1)^{\frac{1}{2}}}
\end{aligned}
$$

As a consequence of (5.5), (5.6) and (5.3) we find that

$$
\bar{\partial}\left(\frac{1}{c_{1}}-c_{2}\right)=0, \quad \bar{\partial}\left(A c_{1} c_{2} \cdots c_{n-1}-c_{1}\right)=0, \text { etc. }
$$

and therefore all the members of the chain (5.3) represent first integrals of the system (5.1) and (5.2):

$$
\frac{1}{c_{k}}-c_{k+1}=y, \quad A c_{1} c_{2} \cdots c_{n-1}-c_{1}=y, \quad \frac{1}{c_{n-1}}-\frac{B}{c_{1} c_{2} \cdots c_{n-1}}=y
$$

where $y=2 i \sin \alpha$ is an arbitrary constant. We next substitute (5.6) and (5.7) in eqns. (5.1) and (5.2) resulting the following set of $2(n+2)$ first order differential equations for $A, B, c_{k}, \tilde{u}$ :

$$
\begin{aligned}
\partial_{\rho_{+}} c_{k} & =2 m \gamma\left(1-c_{k}^{2}-b c_{k}\right), \quad \partial_{\rho_{-}} c_{k}=0 \\
\partial_{\rho_{+}} A & =\frac{2 m \gamma}{\alpha_{0}^{(n-1)}}\left(1-(-1)^{n-1} A^{2}-A\left(\alpha_{0}^{(n-2)}+\alpha_{0}^{(n)}\right)\right), \quad \partial_{\rho_{-}} A=0 \\
\partial_{\rho_{+}} \tilde{u} & =0, \quad \partial_{\rho_{-}} \tilde{u}=-\frac{m y}{2} \tilde{u}
\end{aligned}
$$

where $\alpha_{0}^{(s)}=-i \frac{\sin \left[(s+1)\left(\alpha+\frac{\pi}{2}\right)\right]}{\cos \alpha}$. The equation for $B$ is similar to the one for $A$. The following algebraic relation

$$
B=\frac{(-1)^{n-1} A+\alpha_{0}^{(n)}}{1-\alpha_{0}^{(n-2)} A}
$$

holds. In fact, one has to solve equations for $c_{k}$ only since $A$ and $B$ can be obtained from (5.3). Applying once more the methods used for the axial model (1.1) in Sect. 4, we get the 1-soliton solutions for the vector model (1.3):

$$
\begin{aligned}
c_{k} & =e^{i(\alpha-\pi \operatorname{Sign}(\alpha))} \frac{e^{-f}-(-1)^{k-n} e^{2 i \alpha(n-k-1)} e^{f}}{e^{-f}+(-1)^{k-n} e^{2 i \alpha(n-k)} e^{f}}, \\
A & =e^{-i n(\alpha-\pi \operatorname{Sign}(\alpha))} \frac{e^{-f}+(-1)^{n} e^{2 i \alpha n} e^{f}}{e^{-f}+e^{f}} \\
B & =e^{i n(\alpha-\pi \operatorname{Sign}(\alpha))} \frac{e^{-f}-e^{-2 i \alpha} e^{f}}{e^{-f}+(-1)^{1-n} e^{2 i \alpha(n-1)} e^{f}} \\
\tilde{u} & =e^{-i\left(m \rho_{-} \sin \alpha+q\right)}
\end{aligned}
$$


where $f=\rho_{+} m \cos \alpha+\frac{1}{2} \ln (\delta)$

5.3 Soliton Spectrum. The $U(1)$ symmetry

$$
A^{\prime}=e^{i a \beta_{0}^{2}} A, \quad B^{\prime}=e^{-i a \beta_{0}^{2}} B, \quad c_{k}^{\prime}=e^{\frac{-i a \beta_{0}^{2}}{n}} c_{k}
$$

of $\mathcal{L}_{v}(1.3)$ gives rise to the following "electric" conserved current

$$
J_{e l}^{\mu, v e c}=i\left(\frac{A \partial^{\mu} B-B \partial^{\mu} A}{1-A B}+\partial^{\mu} \ln \left(c_{1} c_{2} \cdots c_{n-1}\right)\right)
$$

As in the axial model $\left(J_{e l}^{\mu, a x}=2 \beta_{0} \epsilon^{\mu \nu} \partial_{\nu} R\right)$ it can be realized in terms on the nonlocal field $u$ (or $\tilde{u}$ ) from eqns. (2.13) and (5.6), i.e. $J_{e l}^{\mu, v e c}=-i \epsilon^{\mu \nu} \partial_{\nu} \ln \left(\tilde{u}^{2}\right)$. The corresponding electric charge

$$
Q_{e l}^{v e c}=\int_{-\infty}^{\infty} J_{e l}^{0, v e c} d x=-i \int_{-\infty}^{\infty} d x \partial_{x} \ln \left(\tilde{u}^{2}\right)
$$

vanishes for the 1-soliton solutions (5.8), i.e. $Q_{e l}^{v e c}=0$. Observe that according to the axial-vector change of variables (2.14) we have

$$
\tilde{u}^{2}=\frac{\psi}{\chi} . \quad \tilde{u}=\frac{u\left(c_{1} c_{2} \cdots c_{n-1}\right)^{\frac{1}{2}}}{(A B-1)^{\frac{1}{2}}}, \quad \tilde{u}=e^{i \beta_{0} \theta}
$$

and therefore the electric current $J_{e l}^{\mu, v e c}$ of the vector gauged model is T-dual (see ref. [15]) of the topological current $J_{\theta}^{\mu, a x}=-\frac{i}{2 \beta_{0}^{2}} \epsilon^{\mu \nu} \partial_{\nu} \ln \left(\frac{\psi}{\chi}\right)=\frac{1}{2 \beta_{0}^{2}} J_{e l}^{\mu, v e c}$ of the axial model (1.1) (remember that $Q_{\theta}^{a x}=0$ for the 1-soliton solutions of the axial model (4.6) and (4.10)). Next step is to recognize that the b.c. (i.e. $\alpha$ ) dependence of the vector model soliton spectrum $\left(M^{v e c}, E^{v e c}, Q_{e l}^{v e c}, Q_{\tilde{\theta}}^{v e c}, Q_{m a g}, s^{v e c}\right)$ comes now from the topological current( for $\cos \alpha>0$ )

$$
J_{\tilde{\theta}}^{\mu, v e c}=-i \epsilon^{\mu \nu} \partial_{\nu} \ln A^{2}, \quad, \quad Q_{\tilde{\theta}}^{v e c}=-i \int_{-\infty}^{\infty} d x \partial_{x} \ln A^{2}=4 n\left(\alpha-\frac{\pi}{2} \operatorname{Sign}(\alpha)\right)+4 \pi j_{\varphi}
$$

It turns out to be T-dual [15] to the axial model electric current $J_{e l}^{\mu, a x}=2 \beta_{0} \epsilon^{\mu \nu} \partial_{\nu} R$. It reflects the fact that the vector model fields $A$ and $B$ have nontrivial asymptotics at $x \rightarrow \pm \infty$,

$$
A(+\infty)=e^{i n \alpha}=\frac{1}{B(+\infty)}, \quad A(-\infty)=e^{-i n(\alpha-\pi \operatorname{Sign}(\alpha))}=\frac{1}{B(-\infty)}
$$

The origin of the symmetries generated by the current $J_{\tilde{\theta}}^{\mu, v e c}$ is similar to one of the axial model (2.20),

$$
A^{\prime}=e^{i \pi s_{1}} A, \quad B^{\prime}=e^{i \pi s_{2}} B, \quad c_{k}^{\prime}=e^{-i \pi \frac{s_{1}}{n}} c_{k}
$$

$s_{1}+s_{2}=2 K, \quad s_{1}-s_{2}=2 L$. One might find contradictory the continuous $\alpha$ dependence of the topological charge $Q_{\tilde{\theta}}^{v e c}$ (5.11). In fact $\alpha$ should be quantized, i.e. $\alpha-\frac{\pi}{2} \operatorname{Sign}(\alpha)=$ $\frac{\beta_{0}^{2}}{4 n}\left(j_{e l}-\frac{4 \pi}{\beta_{0}^{2}} j_{\varphi}\right)$, as we shall see in Sect. 7 . 
The remaining (true) topological charges $\tilde{Q}_{k}$ (i.e. the vector analogs of $Q_{k}^{t o p}, J_{k}^{\mu}=$ $\left.\frac{2 n}{\beta_{0}} \epsilon^{\mu \nu} \partial_{\nu} \varphi_{k}\right)$, can not be defined as $Q_{k}=\int d x \partial_{x} \ln \left(c_{k}\right)$ since the asymptotics of $c_{k}$ include certain $\alpha$ dependence, as one can see from eqn. (5.8),

$$
c_{k}(+\infty)=e^{-i \alpha}, \quad c_{k}(-\infty)=e^{i(\alpha-\pi \operatorname{Sign}(\alpha))}
$$

Taking into account the axial-vector transformation (2.14) and the vector model 1-soliton asymptotics (5.12) and (5.13) it is easy to check that

$$
\left.\frac{i}{\beta_{0}} \ln \left(c_{k} c_{k+1} \cdots c_{n-1} A^{\frac{n-k}{n}}\right)\right|_{ \pm \infty}=\frac{2 \pi}{\beta_{0}} \frac{k}{n} \tilde{N}_{ \pm}, \quad k=1,2, \cdots, n-1
$$

and $\tilde{N}_{ \pm}$are arbitrary integers. Hence we can take

$$
\tilde{Q}_{k}^{t o p}=\frac{2 n}{\beta_{0}} \int_{-\infty}^{+\infty} \partial_{x} \ln \left(c_{k} c_{k+1} \cdots c_{n-1} A^{\frac{n-k}{n}}\right)=\frac{4 k \pi}{\beta_{0}^{2}} j_{\varphi}=Q_{k}^{\text {top }}
$$

It is important to note that although the zeros of the vector model potential (i.e. its vacua) are again labeled by two integers $(\tilde{L}, \tilde{N})$ the vector 1 -solitons $(5.8)$ interpolating between two vacua with both $\tilde{L}$ and $\tilde{N}$ different from zero,$j_{\tilde{\theta}}=\tilde{L}_{+}-\tilde{L}_{-} \neq 0$ (see (5.18)), $j_{\varphi}=\tilde{N}_{+}-\tilde{N}_{-} \neq 0$ contrary to the axial model charged solitons (4.6) and (4.10) that have $Q_{\theta}^{a x} \sim L_{+}-L_{-}=0$, i.e. relates the trivial vacua $(0,0)$ to $(0, N)$ (for $\nu=0$, i.e. without topological $\theta$-term).

The derivation of the 1-solitons energy $E^{v e c}$ and momentum $P^{v e c}$ is similar to the axial case presented in Sect. 3. The main tools are again the soliton conservation laws (5.5) and (5.6) that allow to show that $T_{01}^{v e c}$ and $T_{00}^{v e c}$ are indeed total derivatives. Thus, we find that the $E^{v e c}$ and $P^{v e c}$ are certain functions of the asymptotics of fields $A, B, c_{k}$ (5.12) similar to the axial $E$ and $P$ (3.21) and (3.22) replacing $Q_{e l}^{a x}$ by its dual $Q_{\tilde{\theta}}^{v e c}$,

$$
M^{v e c}=\frac{4 n m}{\beta_{0}^{2}}\left|\sin \frac{1}{4 n}\left(Q_{\tilde{\theta}}^{v e c}-\beta_{0}^{2} Q_{m a g}\right)\right|=M^{a x}
$$

In order to complete the analogy with the axial model soliton spectrum (including $\nu$ and $\tilde{\gamma}$ ) we have to add a purely topological CPT-breaking term to the CPT-invariant vector Lagrangean (1.3) $\mathcal{L}_{\text {impr }}=\mathcal{L}_{\text {vec }}+\delta \mathcal{L}_{\text {top }}^{v}$

$$
\delta \mathcal{L}_{\text {top }}^{v}=\sum_{k=1}^{n-1} \frac{\gamma_{k}}{\beta^{2}} \epsilon^{\rho \nu} \partial_{\rho} \ln \left(c_{k} \cdots c_{n-1}\right) \partial_{\nu} \ln \left(\frac{A}{B}\right)
$$

Although the equations of motion remains unchanged $\delta \mathcal{L}_{\text {top }}^{v}$ contributes to $J_{e l}^{\mu, v e c}$ (i.e. to $\left.J_{\theta}^{\mu, a x}\right)$ as it was explained in Sect.2.5 :

$$
\begin{aligned}
& Q_{e l, i m p r}^{v e c}=\beta_{0} \int_{-\infty}^{\infty} \partial_{x} \theta_{i m p r} d x=Q_{\theta}^{a x}+4 \pi \tilde{\gamma} j_{\varphi}=Q_{\theta, i m p r}^{a x} \\
& Q_{\tilde{\theta}, i m p r}^{v e c}=\beta_{0} \int_{-\infty}^{\infty} \partial_{x} \tilde{\theta}_{i m p r} d x=Q_{e l}^{a x}-\frac{\nu \beta_{0}^{2}}{2 \pi} j_{\varphi}
\end{aligned}
$$


Taking into account that $\frac{Q_{\hat{\theta}, i m p r}^{v e c}}{\beta_{0}^{2}}$ is a topological charge, i.e.

$$
\frac{2 \pi}{\beta_{0}^{2}} Q_{\tilde{\theta}, i m p r}^{v e c}=2 \pi j_{\tilde{\theta}}, \quad j_{\tilde{\theta}}=0, \pm 1, \pm 2, \cdots
$$

we find

$$
Q_{\tilde{\theta}}^{v e c}=\beta_{0}^{2}\left(j_{\tilde{\theta}}+\frac{\nu}{2 \pi} j_{\varphi}\right)=Q_{e l}^{a x}\left(j_{e l} \rightarrow j_{\tilde{\theta}}\right)
$$

The complete discussion of the T-duality relations between the solitons and breathers spectrum of the axial and vector models requires further investigation.

\section{$6 \quad$ Soliton Spin}

6.1 Weak coupling spectrum. The fields (and particles) in 2-d relativistic theories belong to certain representations of the 2 -d Poincare group, $\mathcal{P}_{2}=O(1,1) \otimes T_{2}$. As one can see from its algebra:

$$
\left[M_{01}, P^{ \pm}\right]= \pm P^{ \pm}, \quad\left[P^{+}, P^{-}\right]=0
$$

the Lorentz boosts $M_{01}$ and the mass operator $2 P^{+} P^{-}$provide a set of mutually commuting operators. Their eigenvalues $\left(s, M^{2}\right)$ are used to characterize the $\mathcal{P}_{2}$ representations. For example, the field $\phi_{(s)}(z, \bar{z})$ of Lorentz spin $s$ tranforms under $O(1,1)$ Lorentz rotations : $z^{\prime}=e^{\delta} z, \bar{z}^{\prime}=e^{-\delta} \bar{z}(\delta$ real $)$ as

$$
\phi_{(s)}^{\prime}(z, \bar{z})=e^{\delta M_{01}} \phi e^{-\delta M_{01}}=e^{s \delta} \phi_{(s)}\left(z^{\prime}, \bar{z}^{\prime}\right)
$$

or infinitesimaly,

$$
\left[M_{01}, \phi_{(s)}(z, \bar{z})\right]=(z \partial-\bar{z} \bar{\partial}+s) \phi_{(s)}(z, \bar{z})
$$

In $\mathcal{P}_{2}$ invariant theories $M_{01}$ appears as $O(1,1)$ Noether charge in the well known form $i M_{01}=\int_{-\infty}^{\infty} x T_{00} d x$. Taking the explicit form of $T_{00}$ (say, (3.15)) in terms of fields and their

momenta and using the canonical commutation relations it is straightfoward to derive the canonical spins of all fields:

$$
s_{\varphi_{l}}=0, \quad s_{\psi}=-s_{\chi}=\frac{(n-1)}{2}
$$

Similarly we find for their $U(1)$ charges

$$
Q_{e l}^{\varphi_{l}}=0, \quad Q_{e l}^{\psi}=-Q_{e l}^{\chi}=\beta_{0}^{2}
$$

It is instructive to write eqns. (6.2), (6.3) in a compact form, relating spins with electric charges:

$$
s=\frac{(n-1)}{2 \beta_{0}^{2}} Q_{e l}
$$


We complete the discussion of the spectrum of "weak coupling" $\left(\beta_{0}^{2} \rightarrow 0\right)$ fields (particles) by noting that the "bare" masses of the "weak" fields of the $A_{n}^{(1)}$ model (1.1) are given by

$$
m_{\psi}=m_{\chi}=m, \quad m_{\varphi_{l}}=2 m \sin \left(\frac{\pi l}{n}\right)
$$

6.2 Strong coupling particles. The main characteristic of the "weak" coupling particles is that they do not carry topological charges, $Q_{\theta}=0, Q_{l}^{\text {top }}=0$, as one can easily check by substituting the weak coupling "free" solutions into (2.25) and (2.26). It reflects the fact that all "weak" fields have vanishing assymptotics at $x \rightarrow \pm \infty$. The analysis of the classical vacua structure (i.e. nontrivial constant solutions) of the axial $A_{n}^{(1)}$ model presented in Sect. 2.3 allows to list all the addmissible boundary conditions

$$
\varphi_{l}^{(N)}( \pm \infty)=\frac{2 \pi}{\beta_{0}} \frac{l N_{ \pm}}{n}, \quad r( \pm \infty)=0, \quad \theta^{(L)}( \pm \infty)=\frac{\pi L_{ \pm}}{2 \beta_{0}}
$$

The solutions of eqns. (2.1) with nonvanishing asymptotics (6.6) such that $j_{\varphi}=N_{+}-N_{-} \neq 0$ and/or $j_{\theta}=L_{+}-L_{-} \neq 0$ are by construction topologically charged. The simplest example $j_{\varphi}=1, j_{\theta}=0$ of finite energy electrically charged topological soliton is given by eqns. (4.6) and (4.10). As it is expected its semiclassical spectrum (1.5) shows the characteristic "strong coupling" dependence on the coupling constant $\beta_{0}^{2}$. They define a "strong coupling" set of particles labled by $\left(M, s ; Q_{e l}, Q_{m a g}, Q_{\theta}\right)$. The knowledge of the explicit values of these conserved charges (classical, semiclassical and quantum ) is crucial in answering the question about "strong coupling" symmetry group of the model as well as in the construction of the dual model in terms of the "strong fields" (carrying the strong coupling particles quantum numbers ). It is clear that the spin of the strong fields is the main ingredient in writing the kinetic part of the S-dual Lagrangean.

6.3 Lorentz spin of electrically charged topological solitons. As we have shown in Sect. 3.2, the fact that 1-soliton stress-tensor is a total derivative

$$
T_{00}=\partial_{x}\left(F^{-}-F^{+}\right), \quad T_{01}=\partial_{x}\left(F^{-}+F^{+}\right)
$$

is crucial in the demonstration that solitons energy (and mass) get contributions from the boundary terms only. One expects that similar arguments take place in the calculation of the soliton spin. What we need in this case is to represent $T_{00}$ (and $T_{01}$ ) as certain second derivatives

$$
T_{00}=-\frac{2}{\beta} \partial_{x}^{2}\left(\sum_{k=1}^{n-1} \varphi_{k}+\frac{n-1}{2} R+n \zeta\right),
$$

An auxiliary field $\zeta$ we have introduced in (6.7) is defined as solution of the following first order equations:

$$
\begin{aligned}
\partial \zeta & =\frac{m}{\beta} \gamma e^{\beta\left(\varphi_{n-1}+\frac{R}{n}\right)} \\
\bar{\partial} \zeta & =-\frac{m}{\beta \gamma}\left(e^{\beta\left(\varphi_{1}-\frac{R}{n}\right)}+\beta^{2} \psi \chi e^{-\beta \frac{R}{n}}\right)
\end{aligned}
$$


As a consequence of (6.8) and eqns. (3.7)-(3.8) we realize that $\partial \bar{\partial} \zeta$ is proportional to the trace $T_{\mu}^{\mu}$ of the stress-tensor $T_{\mu \nu}$ 巴马 :

$$
\partial \bar{\partial} \tilde{\zeta}=T_{\mu}^{\mu}=\frac{m^{2}}{\beta}\left(e^{\beta\left(\varphi_{1}+\varphi_{n-1}\right)}+\beta^{2} \psi \chi e^{-\beta \varphi_{n-1}}\right), \quad \tilde{\zeta}=\zeta+\frac{m^{2}}{\beta} z \bar{z}
$$

The proof of eqn. (6.7) is based on the following identity

$$
\partial_{x}\left(\sum_{k=1}^{n-1} \varphi_{k}+\frac{n-1}{2} R+n \zeta\right)=\frac{\beta}{2}\left(F^{+}-F^{-}\right)
$$

which can be easely checked taking into account eqns. (3.7)-(3.8) and (6.8). With the explicit 1-soliton solutions (4.5)-(4.7) at hand we find that $\zeta$ satisfying eqns. (6.8) has the form

$$
\zeta=\frac{1}{\beta} \ln \left(1+e^{2 f}\right)-2 \frac{m}{\beta}(\cos \alpha) \rho_{+}+2 i \frac{m}{\beta}(\sin \alpha) \rho_{-}
$$

Note that for imaginary coupling $\beta=i \beta_{0}$ the stress tensor is complex, say for $T_{00}$ we have

$$
T_{00}=\frac{2}{\beta_{o}^{2}} \partial_{x}^{2} \tau, \quad \tau=i \beta_{0}\left(\sum_{i=1}^{n-1} \varphi_{k}+\frac{n-1}{2} R+n \zeta\right)
$$

Although the energy (3.21) of the 1-solitons is real and finite, their spin $M_{01}$ is in general complex and also contains an infinite part

$$
M_{01}=-i \int_{-\infty}^{\infty} x T_{00} d x=-\left.\frac{2 i}{\beta_{0}^{2}}\left(x \partial_{x} \tau-\tau\right)\right|_{-\infty} ^{\infty}
$$

Its real part, however is finite

$$
s=\operatorname{Re}\left(M_{01}\right)=\frac{(n-1)}{2 \beta_{0}^{2}}\left(Q_{e l}^{a x}+\beta_{0}^{2} Q_{m a g}\right)
$$

and we take it as a definition of the spins of 1-solitons in consideration. It is worthwhile to mention that eqns. (6.7)- 6.13$)$ with $R=0,\left(Q_{e l}=0\right)$ remain valid for the solutions of the $A_{n-1}^{(1)}$-abelian affine Toda, i.e. the spins of the 1-solitons of this model turns out to be

$$
s_{\text {Ab.Toda }}=\frac{n-1}{2} Q_{m a g}
$$

\section{Towards Exact Quantization}

7.1 Semiclassical Quantization. Classical periodic motions are known to correspond to quantum bound states (discrete energy spectrum $E_{j}$ ) such that the frequency of the emition

\footnotetext{
${ }^{13}$ the field $\tilde{\zeta}$ with the above properties together with the free field $\eta$ are familiar from the conformal affine extension [19] of the affine NA-Toda models
} 
$E_{j} \rightarrow E_{j+1}$ coincides with the frequency of the classical motion. The energy eigenvalues $E_{j}$ can be found from the Bohr-Sommerfeld quantization rule $\int_{0}^{\tau} d t p \dot{q}=2 \pi j$ ( $j$ an integer ), i.e.

$$
E_{j} \tau+S=2 \pi j, \quad S=\int_{0}^{\tau} d t \mathcal{L}
$$

As we have shown in Sect. 4 (see eqn. (4.12)) our charged 1-solitons (4.6), (4.10) at the rest frame $v=\tanh (b)=0$ represent periodic particle-like motion $\tau=\frac{2 \pi}{m \sin \alpha}$ similar to the SG breather. Hence, the semiclassical soliton spectrum can be derived from the field theory analog of eqn. (7.1)

$$
\begin{aligned}
& \mathcal{H}=\Pi_{\rho_{i}} \dot{\rho}_{i}-\mathcal{L}, \quad \Pi_{\rho_{i}}=\frac{\delta \mathcal{L}}{\delta \dot{\rho}_{i}}, \quad \rho_{i}=\left(\psi, \chi, \varphi_{k}\right) \\
& S+E(v=0) \tau=\int_{0}^{\tau} \int_{-\infty}^{\infty} \Pi_{\rho_{i}} \dot{\rho}_{i}=2 \pi j_{e l}
\end{aligned}
$$

Taking into account the relations between $\psi \Pi_{\psi}-\chi \Pi_{\chi}$ and $\partial_{x} R$ we realize that

$$
\Pi_{\psi} \dot{\psi}+\Pi_{\chi} \dot{\chi}=-\frac{i}{\beta_{0}} \partial_{x} R \partial_{t} \ln \left(\frac{\chi}{\psi}\right)+\frac{i}{\beta_{0}} \partial_{t} R \partial_{t} \ln (\chi \psi)
$$

At the rest frame $(\cosh b=1, \sinh b=0)$ our 1-soliton solutions (4.6), (4.7) and (4.9) have a simple t-dependence:

$$
\partial_{t} \ln \left(\frac{\chi}{\psi}\right)=2 i m \sin \alpha, \quad \partial_{t} \ln \psi \chi=0, \quad \partial_{t} \varphi_{i}=\partial_{t} \Pi_{\varphi_{i}}=0
$$

Therefore we find (for $\nu=0$ )

$$
\int_{0}^{\tau} \int_{-\infty}^{\infty} \Pi_{\rho_{i}} \dot{\rho}_{i}=\frac{2 \pi}{\beta_{0}^{2}} Q_{e l}^{a x}
$$

In the case $\nu \neq 0$ the corresponding momenta of the fields $\Pi_{\rho_{i}}$ acquire improvements resulting in

$$
\int_{0}^{\tau} \int_{-\infty}^{\infty} \Pi_{\rho_{i}} \dot{\rho}_{i}=\frac{2 \pi}{\beta_{0}^{2}} \int_{-\infty}^{\infty} J_{e l, i m p r}^{0, a x} d x
$$

This leads to the following semiclassical quantization of $Q_{e l}$ (see eqn. (2.29) for the definition of $\left.J_{\text {el,impr }}^{0, a x}\right)$ :

$$
Q_{e l}^{a x}=\beta_{0}^{2}\left(\frac{\nu}{2 \pi} j_{\varphi}+j_{e l}\right)
$$

We find instructive to present an alternative derivation of the quantization rule (7.2), (7.3) by explicit calculation of the action $S(\tau)=\int_{0}^{\tau} \int_{-\infty}^{\infty} d x \mathcal{L}_{a}$ on the 1-soliton solutions. The key point is to demonstrate that $\mathcal{L}_{a}$ is a total derivative due to the first order equations (3.7) and (3.10) and the soliton conservation laws (3.11), (3.12) and (3.13). The calculation 
is a bit more involved than the similar one in (3.15) concerning $T_{00}$ and $T_{01}$. That is why we consider the simplest case of $A_{2}^{(1)}$ only $(\gamma=1, \cosh (b)=1, v=0, \nu=0)$

$$
\mathcal{L}_{a}=\partial \varphi \bar{\partial} \varphi+\frac{\bar{\partial} \psi \partial \chi}{\Delta} e^{-\beta \varphi}-\beta^{2} V_{a}=-T_{00}+\frac{2 m}{\beta} \sin \alpha \partial_{x} R
$$

where we have used

$$
\partial R=\frac{2}{y \beta}\left(e^{-\beta R}+e^{\beta R}-2-y^{2}\right), \quad \bar{\partial} R=-\frac{2}{y \beta}\left(e^{-\beta R}+e^{\beta R}-2-y^{2}\right)
$$

Therefore we have,

$$
S_{M}(\tau)=-\tau E(v=0)+\frac{2 \pi}{\beta_{0}^{2}} Q_{e l}^{a x},
$$

that together with $(7.2)$ leads again to $(7.3)$. It is important to note that repeating this calculation for the Euclidean case $t \rightarrow i t$ we find

$$
S_{E}(\tau)=\frac{2 \pi}{\beta_{0}^{2}} Q_{e l}^{a x}
$$

i.e. the Euclidean charged 1-solitons (they are not static ) have properties similar to the instantons, i.e. the Euclidean action is bounded below by the electric charge. In the case of the vector gauged model (1.3) all the results have the same form as above with $Q_{e l}^{a x} \rightarrow Q_{\tilde{\theta}}^{v e c}$.

7.2 Exact Counterterms and $\beta$ renormalization. The quantum properties of the $A_{n}^{(1)}$ dyonic integrable modes (1.1) and (1.3) turns out to be quite similar to the CSG model [12]. While the main effect of the soliton quantization of the SG and $A_{n}^{(1)}$ abelian affine Toda theories is the coupling constant renormalization [24, 31] in the CSG, as well as in the models in consideration the 1-loop calculation shows that new counterterms are needed in order to define a consistent quantum theory.

The same phenomenon takes place in the "free limit" $V=0$ ( i.e. $m=0$ ) of (1.1) and (1.3) known to describe strings in curved background: 2-D blackhole $(n=1), 3$-D black string $(n=2)$, etc [27. Since they can be realized as gauged $\frac{S L(2) \otimes U(1)^{n-1}}{U(1)}$ WZW models, the best way 14 of deriving the corresponding quantum theories (i.e. renormalization, counterterms, etc ) is by applying the functional integral methods [23]. The path integral formalism for the conformal limit of our dyonic models (i.e. $V_{\text {def }}=0$ in (1.2) ) 14 was extended in our recent paper 222 to the case of a large family of integrable models, that can be represented as two loop gauged WZW model $\hat{H}_{-} \backslash G_{n}^{(1)} / \hat{H}_{+}=G_{0} / U(1), \quad G_{0}=S L(2) \otimes U(1)^{n-1}$. Integrating over the infinite $\hat{H}_{ \pm}$but keeping $U(1)$ (spanned by $\lambda_{1} \cdot H^{(0)}$ ) ungauged, we find the following effective action (of the intermediate model (1.4) for $A_{0}=\bar{A}_{0}=0$ )

$$
\begin{aligned}
S_{\text {eff }}^{G_{0}} & =S_{W Z W}^{G_{0}}\left(D_{v}\right)-\frac{1}{\beta_{0, r e n}^{2}} \int d x^{2} \operatorname{Tr}\left(\epsilon_{+} D_{v} \epsilon_{-} D_{v}^{-1}\right) \\
& +\frac{1}{\beta_{0, r e n}^{2}} \int d x^{2} \operatorname{Tr}\left(A_{0} \bar{\partial} D_{v} D_{v}^{-1}-\bar{A}_{0} D_{v}^{-1} \partial D_{v}-D_{v}^{-1} A_{0} D_{v} \bar{A}_{0}+\left(1+\lambda_{0}\right) A_{0} \bar{A}_{0}\right)
\end{aligned}
$$

\footnotetext{
14 an alternative way is to use the representation theory of the parafermionic extensions of the Virasoro algebra. The quantum theory of the conformal " non free" $A_{2}^{(1)}$ NA-Toda model is shown to be equivalent to the representation theory of the $V_{3}^{(1,1)}$-algebra 14]
} 
Extending Tseytlin's arguments (see Sect. 3 and 4 of the first of refs (23)) to the $S_{\text {eff }}^{G}$, given by (7.6), we realize that

$$
\begin{aligned}
\beta_{0, \text { ren }}^{2} & =\frac{2 \pi}{k-n-1}, \quad \beta_{0}^{2}=\frac{2 \pi}{k} \\
\lambda_{0} & =2 \frac{n+1}{k-n-1}=\frac{(n+1)}{\pi} \beta_{0, \text { ren }}^{2}
\end{aligned}
$$

( and $1+\lambda_{0}=1$, i.e. $\lambda_{0}=0$ is the unrenormalized "classical" coefficient of $A_{0} \bar{A}_{0}$ term responsible for the cancellation of the $U(1)$ gauge anomaly). Integrating out $A_{0}, \bar{A}_{0}$ in the partition function

$$
Z_{A_{n}^{(1)}}^{v}=\int \mathcal{D} A_{0} \mathcal{D} \bar{A}_{0} \mathcal{D} D_{v} e^{-S_{\text {eff }}^{G_{0}}}=N_{\infty} \int \mathcal{D} D_{v} e^{-S_{\text {eff }}^{G_{o} / U(1)}}
$$

we find the renormalized quantum action for the dyonic model (1.3)

$$
\left.S_{e f f}^{G_{0} / U(1)}=\int \mathcal{L}_{v} d^{2} x+\frac{n+1}{8 \pi} \int \frac{\left(A \partial_{\mu} B-B \partial_{\mu} A\right)^{2}}{(1-A B)\left(1+\lambda_{0}-A B\right)}+i \int \frac{R^{(2)}}{\beta_{0}} \sum_{k=1}^{n} \beta_{k} \ln \left(c_{k}\right) d^{2} x\right)
$$

It differs from the classical action (1.3) by

a) the counterterm

$$
\mathcal{L}_{c . t .}=\frac{n+1}{8 \pi} \frac{\left(A \partial_{\mu} B-B \partial_{\mu} A\right)^{2}}{(1-A B)\left(1+\lambda_{0}-A B\right)},
$$

b) the dilaton contribution

$$
\mathcal{L}_{d i l}=\frac{i}{\beta_{0}} R^{(2)} \sum_{k=1}^{n} \beta_{k} \ln c_{k}, \quad \ln c_{n}=\frac{i \beta_{o}}{2} \ln (1-A B), \quad \beta_{1}=-3, \beta_{k}=-1, k=2, \cdots, n
$$

( $R^{(2)}$ is the worldsheet curvature).

c) the $\beta$ renormalization (7.7).

Expanding denominators in (7.9) we find it consistent with the 1-loop (in $\beta_{0, r e n}^{2}$ ) result (see ref. 12 for $n=1$ case and 32 for the $B_{n}^{(1)}$ T-self dual Fateev's models ).

7.3 $U_{q}(\hat{S L}(n+1))$ Symmetries of the Soliton Spectrum. The most important question concerning the quantum solitons and breathers spectrum is about the solitons symmetry group. Following the paralel with the $A_{n}^{(1)}$ abelian affine Toda models where the quantum solitons are related to the representations with $q^{k+n+1}=1$ of the affine quantum group $U_{q}(\hat{S L}(n+1))$ [8] one expects that neutral and charged 1-solitons of our dyonic models to have similar properties whether it is true is an open question, although there exist few hints that this is indeed the case:

- Neutral 1-solitons and their conservation laws coincides with the $A_{n-1}^{(1)}$ abelian affine Toda solitons, that is why neutral sector should manifest $U_{q}(\hat{S L}(n))$ symmetry (but not $\hat{S L}(n+1, q))$. 
- The form of the classical conservation laws (3.11) for the charged solitons is similar to the abelian case and the classical "braiding relations" of the currents components encoded in the classical $r$-matrix [39 are supporting the conjecture the $U_{q}(\hat{S L}(n+1))$ is the charged solitons group of symmetries.

- The $n=1$ case (i.e. the quantun CSG model ) corresponds to certain thermal perturbations of the parafermionic conformal models [33]. Its quantum S-matrix confirms the $U_{q}(\hat{S L}(2))$ symmetry in this case.

- The $n=2$ (i.e. $A_{2}^{(1)}$ ) dyonic model can be realized as an appropriate perturbation of the $V_{3}^{(1,1)}$-algebra conformal minimal models constructed in [14] (see Sect. 9 of [14]). Our preliminary calculation based on bosonization of the vertices representing quantum solitons shows that the $A_{2}^{(1)}$ dyonic models possess a nontrivial set of quantum conserved currents, whose charges span the $S L(3)_{q}$ quantum affine algebra.

\section{Concluding Remarks}

8.1 S-duality. The particle-like nonperturbative solutions of $S U(n+1)$ YMH modelmonopoles and dyons are believed to provide the fields degrees of freedom relevant for the description of the strong coupling phase of YMH (and of QCD in general ). Similar phenomena is known to take place in 2-D IM: Topological solitons serve as strong coupling variables dual to the fundamental (weak coupling) particles (fields), presented in the IM's " weak coupling " actions (say, (1.1)) [34, 32]. The simplest and best understood example is the strong-weak coupling (S-) duality between the massive Thirring and the sine-Gordon models [34, 35.

The knowledge of the exact quantum dyons spectrum is crucial in the derivation of the YMH model strong coupling symmetry group and its representations to be used in the construction of the strong coupling YMH effective action. The Montonen-Olive duality conjecture [36] states that the monopoles of the $G_{n^{-}}$YMH model belongs to fundamental representations of the dual group $G_{n}^{v}$ (say, $G_{n}=S U(n+1)$, and $\left.G_{n}^{v}=S U(n+1) / Z_{n+1}\right)$. It has been proved for $N=4$ SUSY $G_{n}$ YM theories and certain $N=2$ SUSY YM-matter models [37].

For $N=1$ and for the non-SUSY YMH models, the exact dyonic spectrum is unknown. As it was argued in the introduction, the study of the $U(1)$ charged topological solitons of appropriate 2-D IM of dyonic type and their exact quantization may contribute to the understanding of the nature of strong coupling symmetry groups of non SUSY YMH theory. The conjectured form of the exact quantum 2-D dyons spectrum and the arguments presented in sect.7.3 makes feasible the role of the centerless quantum affine group $A_{n}^{(1)}(q),(q=$ $\left.e^{i \hbar \beta_{0, \text { ren }}^{2}}, \quad \beta_{0, \text { ren }}^{2}=\frac{2 \pi}{K-n-1}\right)$ as strong coupling symmetry group of the $A_{n}^{(1)}$-dyonic IM's (1.1) and (1.3). The question of whether certain affine quantum group appear in the description of the 4-D YMH quantum dyons and domain walls is far from being answered. The observation concerning the affine $\hat{S U}(n+1)$ symmetries of the classical solutions of the $S U(n+1)$ self dual YM (and the YMH-Bogomolny) equations [9] is an indication that their finite energy quantum solutions could have the $U_{q}(\hat{S U}(n+1))=U_{q}\left(A_{n}^{(1)}\right)$ as an algebra of symmetries. 
The problem to be solved before any attempt for affine quantum group improvement of the original Montonen-Olive conjecture is about the integrable models S-dual to the $A_{n}^{(1)}$ dyonic models (1.1) and (1.3). The fundamental fields used in writing their Lagrangeans have to carry the quantum numbers $\left(Q_{e l}, Q_{m}, M, s, \lambda_{j}, \cdots\right)$ of charged and neutral solitons of the $A_{n}^{(1)}$ models. Its soliton (i.e. strong coupling ) spectrum should have the quantum numbers of the fundamental fields of the dyonic models i.e. $\psi, \chi, \varphi_{i}$. Similarly to the solitons of all known 2-D integrable models they must have certain affine quantum group structure behind. The most natural candidate is the algebra dual to $A_{n}^{(1)}(q)$ with $\tilde{q}=e^{i \hbar \tilde{\beta}^{2}}, \quad \tilde{\beta}^{2}=\frac{1}{\beta^{2}}$. The partition functions of such pair of S-dual IM's are expected to be related by the S-duality transformations

$$
\tau \longrightarrow \frac{n_{1}+m_{1} \tau}{n_{2}+m_{2} \tau}, \quad \tau=\frac{\nu}{2 \pi}+i \frac{2 \pi}{\beta_{r}^{2}}
$$

where $\left(\begin{array}{ll}n_{1} & m_{1} \\ n_{2} & m_{2}\end{array}\right) \in S L(2, Z)$. We have not a recipe of how to construct the S-dual of a given IM satisfying all the requirements listed above. An important experience in this direction are the families of S-dual pairs of IM introduced and studied by Fateev [32]

$$
\begin{aligned}
B_{n+1}^{(1)}\left(\left\{\chi, \bar{\chi}, \phi_{i}\right\}_{\text {strong }}, \beta^{2}\right) & \rightarrow A_{2 n+1}^{(2)}\left(\left\{\psi, \bar{\psi}, \varphi_{i}\right\}_{\text {weak }}, \frac{4 \pi}{\beta^{2}}\right) \\
A_{2 n}^{(2)}\left(\left\{\chi, \bar{\chi}, \phi_{i}\right\}_{\text {strong }}, \beta^{2}\right) & \rightarrow A_{2 n}^{(2)}\left(\left\{\psi, \bar{\psi}, \varphi_{i}\right\}_{\text {weak }}, \frac{4 \pi}{\beta^{2}}\right) \\
D_{n+1}^{(2)}\left(\left\{\chi, \bar{\chi}, \phi_{i}\right\}_{\text {strong }}, \beta^{2}\right) & \rightarrow C_{n}^{(1)}\left(\left\{\psi, \bar{\psi}, \varphi_{i}\right\}_{\text {weak }}, \frac{4 \pi}{\beta^{2}}\right)
\end{aligned}
$$

The "strong coupling models" $G_{n}^{(a)}$ represent the complex SG (Lund Regge) model $(\chi, \bar{\chi})$ interacting with $G_{n-1}^{(a)}$ abelian affine Toda theories $\left(\phi_{i}\right)$. Their actions are similar to (1.3) and as it is shown in [22] they can be derived following the general Hamiltonian reduction procedure (or from gauged two loop WZW model) discussed in Sect. 2. The explicit form of the $\mathcal{G}_{n}^{(1)}$ valued flat connections depend on the specific choice of the grading operator $Q$, constant elements $\epsilon_{ \pm}$and in the way the chiral $U(1)$ symmetry $\mathcal{G}_{0}^{0}$ is gauged fixed. The "weak coupling models" $\tilde{G}_{n}^{(a)}$ represent massive Thirring fermions $\psi, \bar{\psi}$ interacting with the abelian affine Toda model based on the dual algebra $\mathcal{G}_{n-1}^{v}$ of $\mathcal{G}_{n-1}^{(1)}$, i.e. $B_{n+1}^{(1)}$ is dual to $A_{2 n-1}^{(2)}, D_{n+1}^{(2)}$ is dual to $C_{n}^{(1)}$, etc. It is important to note that only the case of real coupling constant has been considered in ref. [32]. Therefore, the corresponding potentials admit only trivial zero $\phi_{i}=0$ (but not $\phi_{i} \in \frac{2 \pi}{\beta} \Lambda_{\omega}, \Lambda_{\omega}$ is the coroot lattice of $G_{n-1}^{(a)}$ ). As a consequence, their finite energy nonperturbative solutions are the $G_{n}^{(a)}$ analogs of the $U(1)$ charged nontopological solitons of the CSG model and their quantization is known to be related to $U_{q}(S L(2)$ ) ( and not to $G_{n}^{(a)}(q)$ ). This explains the pair of Thirring fermions representing these solitons in the weak coupling model. Whether the methods for quantization of these models, for construction of their exact S-matrix, etc. 32 can be applied to the imaginary coupling constant case is an open question. Due to more complicated soliton spectrum of both models for $\beta \rightarrow i \beta_{0}$ their S-duality for imaginary couplings has to be reconsidered. Although the S-duality is a property of the quantum IM (i.e. counterterms, renormalization, etc. are essential), an 
important problem to be addressed is whether exists an algebraic recipe (the 2-d analog of Montonen-Olive conjecture) that for any given IM $\left\{G_{n}^{(1)}, Q, \epsilon_{ \pm}, \mathcal{G}_{0}^{0}\right\}$ prescribes its S-dual IM (the classical limit only) $\left\{\tilde{G}_{n}^{(1)}, \tilde{Q}, \tilde{\epsilon}_{ \pm}, \tilde{\mathcal{G}}_{0}^{0}\right\}$. The simplest problem to be solved is to find the graded structure behind the "weak coupling "Fateev's models for real $\beta$ and to construct their zero curvature representations. The presence of the Thirring fermions interacting with the abelian Toda bosons is an indication that they are from the family of $G_{n}^{(1) V}$ (the group dual of $G_{n}^{(1)}$ ) non abelian Toda-matter models of higher grade, say $\epsilon_{ \pm}$to be of grade \pm 2 (or some halfinteger grade ) and the physical fields to lie in the lowest grades $|s|<2$, as for the IM's constructed in ref. [38].

8.2 T-duality. As we have mentioned in Sects. 1,2 and 5 the two $A_{n}^{(1)}$ dyonic IM's (1.1) and (1.3) studied in the present paper are T-dual by construction. The abelian Tduality transformation (2.14) and (2.16) is specific for the models with target space metrics admitting one isometric coordinate $\theta=-\frac{i}{2 \beta_{o}} \ln \left(\frac{\tilde{\chi}}{\tilde{\psi}}\right)$ for (1.1) and $\tilde{\theta}=\frac{2}{i \beta_{o}} \ln (A)$ for (1.3), (i.e. our models are invariant under global $U(1), \quad \theta \rightarrow \theta+a, \quad \tilde{\theta} \rightarrow \tilde{\theta}+\tilde{a})$. The particular canonical transformation $\left(\theta, \Pi_{\theta}\right) \rightarrow\left(\tilde{\theta}, \Pi_{\tilde{\theta}}\right)$

$$
\Pi_{\theta}=-\partial_{x} \tilde{\theta}, \quad \Pi_{\tilde{\theta}}=-\partial_{x} \theta
$$

which integrated form is eqn. (2.14), are familiar from the string T-duality [28] relating certain conformal $\sigma$ - models representing different curved string backgrounds of 3-D blackstring type [27]. As it was pointed out in Sect. 2, the free limits $m=0$, i.e. $V=0$ of our dyonic models are nothing but the conformal $\sigma$-models considered in ref. [27] in a specific parametrization ( of Gauss type ) of the $A_{n}$ group elements and without the "counterterm" contributions (7.8) and (7.9) essential for the exactness (in $\beta^{2}$ ) of the "target-space " conformal metrics $\left(g_{i j}, b_{i j}, \phi_{d i l}\right)$. The important difference is that in this $m=0$ case there exists more isometries $\varphi_{i}^{\prime}=\varphi_{i}+a_{i}$, together with $\theta^{\prime}=\theta+a_{\theta}$. Adding the conformal ( and nonconformal ) vertices representing nontrivial (bounded for $\beta \rightarrow i \beta_{0}$ ) potentials results in breaking $U(1)^{n}$ to $U(1)$, i.e. the "free" conformal T-duality group $O(n, n \mid Z)$ is broken to $O(1,1 \mid Z)$. An important and new feature of the non - conformal T-duality specific for the dyonic IM's is the relation between their soliton (and breathers ) solutions and the interchanges $Q_{e l}^{a x} \rightarrow Q_{\tilde{\theta}}^{v e c}$ and $Q_{\theta}^{a x} \rightarrow Q_{e l}^{v e c}$ that are IM generalization of the well known maps between the momenta and winding numbers in the conformal (string ) case. The complete discussion of the abelian T-duality for a large class of $U(1)$ symmetric axial and vector IM of dyonic type including the formal proofs of eqns. (2.14) and (2.16) are presented in our work 15.

Since the T-dual IM's (1.1) and (1.3) are expected to be appropriate symmetry reductions of the 4-D $S U(n+1)$-YMH models and their (charged) topological solitons to be related to certain charged domain walls solutions, the natural question is about the 4-D consequences of the T-duality observed in 2-D soliton spectra (see Sect.5). It is clear that they have to represent specific residual (discrete) gauge symmetries as in the string case [28] reflecting the independence of the strong coupling spectra of the manner of the local (unbroken) $U(1)$ gauge symmetry is fixed.

The problem of the $\mathrm{S}$ and T-dualities of the soliton spectra of 2-D IM's of dyonic type deserves special attention and more complete investigation. 
Acknowledgments We are grateful to FAPESP,UNESP and CNPq for financial support.

\section{References}

[1] R.S. Ward, Phil. Trans. R. Soc. Lon. A315 (1985) 451; Lect. Notes Phys. 280 (1987) 106; Lon. Math. Soc. Lec. Notes Ser. 156 (1990) 246

[2] M.J. Ablowitz, S. Chakravarty and L. Takhtajan, PAM Report 108(1991); 113(1991); Commun. Math. Phys. 158 (1993) 289; T.A. Ivanova and A.D. Popov, Theor and Math. Phys. 102 (1995) 280;

[3] A. N. Leznov and M. V. Saveliev, Group Theoretical Methods for Integration of Nonlinear Dynamical Systems, Progress in Physics, Vol. 15 (1992), Birkhauser Verlag, Basel

[4] N. Ganoulis, P. Goddard, D. Olive Nucl. Phys. B205,(1982), 601

[5] E. Witten, Phys. Rev. Lett. 38 (1977) 121

[6] A. Kovner, M. Shiffman and A. Smilga, Phys. Rev. D56, (1997),7978-7989, also G. Dvali and M. Shiffman, Phys. Lett. B396,(1997), hep-th/9612128; A. Hanany and K. Hori, Nucl. Phys. B513 (1998) 119 hep-th/9706089; E. Witten, Nucl. Phys. B507 (1997) 658

[7] J. Gauntlett, D. Tong, P. Townsend, "Supersymmetric intersecting domain walls in massive hyperkahler sigma models", DAMTP-2000-69, hep-th/0007124; J. Gauntlett, R. Portugues, D. Tong, P. Townsend, "D-brane solitons in supersymmetric models", DAMTP-2000-68, hep-th/0008221;

[8] D. Bernard and A. LeClair, Commun. Math. Phys. 142 (1991) 99

[9] L.Dolan,Phys. Reports 109 (1984) 1; Ling-Lie Chau, Ge Mo-Lin and Wu Yong-Shi, Phys. Rev. D25 (1982) 1086

[10] F. Lund, Phys. Rev. Lett. 38 (1977) 1175; B.S. Getmanov, JETP Lett. 25(1977)119

[11] N. Dorey and T.J. Hollowood, Nucl. Phys. B440 (1995) 215

[12] H.J. de Vega and J.M. Maillet, Phys. Rev. D28 (1983) 1441

[13] C.R. Fernandez-Pousa, M.V. Gallas, T.J. Hollowood and J.L. Miramontes, Nucl. Phys. B484 (1997) 609; Nucl. Phys. B499 (1997) 673; C.R. Fernandez-Pousa and J.L. Miramontes, Nucl. Phys. B518 (1998) 745

[14] J.F. Gomes, G.M. Sotkov and A.H. Zimerman, Ann. of Phys.274(1999)289, hepth/9803234; Phys. Lett. 435B (1998) 49, hepth/9803112, 
[15] J.F. Gomes, E.P. Gueuvoghlanian, G.M. Sotkov and A.H. Zimerman, "Torsionless T Selfdual Affine Non Abelian Toda Models", , hep-th/0002173, To appear in the Proc. of the VI International Wigner Symposium, Istambul, Turkey, 2000; see also J.F. Gomes, E.P. Gueuvoghlanian, G.M. Sotkov and A.H. Zimerman, "T-duality of axial and vector dyonic integrable models", hep-th/0007116, to appear in Ann. Phys, (2001)

[16] A.M. Polyakov, Int. J. Mod. Phys. A5(1990)833; M. Bershadsky, Commun. Math. Phys. 139 (1992) 71

[17] D. Olive, N. Turok and J. Underwood Nucl. Phys. B409 (1993) 509; Nucl. Phys. B401 (1993) 663

[18] O. Babelon and D. Bernard, Int. J. Mod. Phys. A8(1993)507; Commun. Math. Phys. 149 (1992) 297

[19] L.A. Ferreira, J.L. Miramontes and J.S. Guillen, Nucl. Phys. B449 (1995) 631

[20] L. Alvarez-Gaumè and F. Zamora, " Duality in Quantum Field Theory (and String Theory)", hepth/9709180, Workshop on Fundamental particles and Interactions, Vanderbilt University and CERN-La-Plata-Santiago-de-Compostela School of Physics, May 1997

[21] J. Gauntlett, N. Kim, J. Park, P. Yi, Phys. Rev. D61 (2000) 125012

[22] J.F. Gomes, E.P. Gueuvoghlanian, G.M. Sotkov and A.H. Zimerman, "Dyonic Integrable Models", hepth/0011187, to appear in Nucl. Phys. B, (2001)

[23] A. A. Tseytlin, Nucl. Phys. B399 (1993) 601; Nucl. Phys. B411 (1994) 509

[24] T.J. Hollowood, Nucl. Phys. B384 (1992) 523

[25] H.C. Liao, D. Olive and N. Turok, Phys. Lett. 298B (1993) 95

[26] H. Aratyn, L.A. Ferreira, J.F. Gomes and A.H. Zimerman, Phys. Lett B 254 (1991) 372; L.A. Ferreira, J.F. Gomes, A. Schwimmer and A.H. Zimerman, Phys. Lett. 274B (1992) 65

[27] J.H. Horn and G.T. Horowitz, Nucl. Phys. B368 (1992) 444; P. Ginsparg and F. Quevedo, Nucl. Phys. B385 (1992) 527

[28] A. Giveon, M. Porrati and E. Rabinovici, Phys. Reports 244 (1994) 77;E. Alvarez, L. Alvarez-Gaumè and Y. Lozano, Nucl. Phys. Proc. Suppl. 41(1995)1

[29] E. Witten, Phys. Lett. 86B (1979) 283

[30] J. Polchinski, String Theory, Vol. I, Cambridge Univ. Press (1998)

[31] R.C. Dashen, B. Hasslacher and A. Neveu, Phys. Rev. D10 (1974) 4114; Phys. Rev. D10 (1974) 4130; Phys. Rev. D10 (1974) 4138; Phys. Rev. D11 (1975) 3424 
[32] V.A. Fateev, Nucl. Phys. B479 (1996) 594

[33] V.A. Fateev and H. deVega, J. Physics A25 (1992) 2693, V. Fateev, Int. J. Mod. Phys. A6 (1991) 2109

[34] S. Coleman, Phys. Rev. D11 (1975) 2088

[35] S. Mandelstam, Phys. Rev. D11 (1975) 3026

[36] C. Montonen and D. Olive, Phys. Lett. 72B (1977) 117

[37] C. Vafa and E. Witten, Nucl. Phys. B431 (1994) 3; N. Seiberg and E. Witten, Nucl. Phys. B426 (1994) 16; Nucl. Phys. B431 (1994) 484

[38] L.A. Ferreira, J-L Gervais, J. Sanchez-Guillen and M.V. Saveliev, Nucl. Phys. B470 (1996) 236

[39] J.F. Gomes, E.P. Gueuvoghlanian, G.M. Sotkov and A.H. Zimerman, "Classical Integrability of Non Abelian Affine Toda Models, to appear in the Proc. of the XXIII International Colloquium on Group Theoretical Methods in Physics, Ed. G. Pogosyan et. al., Dubna (2000), hepth/0010257 